\title{
Extensive nuclear reprogramming and endoreduplication in mature leaf during floral induction
}

\author{
Stefania Del Prete ${ }^{1}$, Anne Molitor ${ }^{1}$, Delphine Charif ${ }^{1}$, Nadia Bessoltane ${ }^{1}$, Ludivine Soubigou-Taconnat ${ }^{2,3}$, \\ Cécile Guichard ${ }^{2,3}$, Véronique Brunaud ${ }^{2,3}$, Fabienne Granier ${ }^{1}$, Paul Fransz ${ }^{4}$ and Valérie Gaudin ${ }^{1 *}$ (D)
}

\begin{abstract}
Background: The floral transition is a complex developmental event, fine-tuned by various environmental and endogenous cues to ensure the success of offspring production. Leaves are key organs in sensing floral inductive signals, such as a change in light regime, and in the production of the mobile florigen. CONSTANS and FLOWERING LOCUS T are major players in leaves in response to photoperiod. Morphological and molecular events during the floral transition have been intensively studied in the shoot apical meristem. To better understand the concomitant processes in leaves, which are less described, we investigated the nuclear changes in fully developed leaves during the time course of the floral transition.
\end{abstract}

Results: We highlighted new putative regulatory candidates of flowering in leaves. We observed differential expression profiles of genes related to cellular, hormonal and metabolic actions, but also of genes encoding long non-coding RNAs and new natural antisense transcripts. In addition, we detected a significant increase in ploidy level during the floral transition, indicating endoreduplication.

Conclusions: Our data indicate that differentiated mature leaves, possess physiological plasticity and undergo extensive nuclear reprogramming during the floral transition. The dynamic events point at functionally related networks of transcription factors and novel regulatory motifs, but also complex hormonal and metabolic changes.

Keywords: Floral transition, Leaf, Arabidopsis, Transcription, Non-coding RNA, Transcription factors, DNA motif, Endoreduplication

\section{Background}

The transition to flowering is a decisive developmental event in the plant life cycle for reproductive success. The general understanding highlights a fine-tuned process involving a complex interplay between environmental and endogenous cues. Signals are perceived and decoded according to the plants' lifestyle, and lead to a cascade of dramatic morphological changes at the meristem level, to produce floral organs $[1,2]$.

Photoperiod is a major parameter controlling the transition to flowering with intricate phototropic effects and links with the circadian clock. The light signal, perceived

\footnotetext{
* Correspondence: valerie.gaudin@inra.fr

${ }^{1}$ Institut Jean-Pierre Bourgin, INRA, AgroParisTech, CNRS, Université Paris-Saclay, INRA Centre de Versailles-Grignon, Bât. 2, RD10 Route de Saint-Cyr, 78000 Versailles, France

Full list of author information is available at the end of the article
}

in the leaves, triggers the accumulation of metabolites and regulators, such as the well-conserved FLOWERING LOCUS T (FT) protein, whose expression is under the control of the CONSTANS (CO), a zinc finger transcription factor involved in photoperiod pathway [3]. Their export, as systemic florigen signals via the vasculature to the distant shoot apical meristem (SAM), activates floral homeotic genes [4-6]. Described as a quantitative long-day (LD) species, the photoperiodic property of Arabidopsis species was exploited to induce synchronous flowering by exposure to a single LD or a single displaced short-day (SD), providing a convenient experimental inductive system [7]. Besides photoperiod, other regulatory pathways partake to the vegetative-to-reproductive switch control [8-10].

(c) The Author(s). 2019 Open Access This article is distributed under the terms of the Creative Commons Attribution 4.0 International License (http://creativecommons.org/licenses/by/4.0/), which permits unrestricted use, distribution, and reproduction in any medium, provided you give appropriate credit to the original author(s) and the source, provide a link to the Creative Commons license, and indicate if changes were made. The Creative Commons Public Domain Dedication waiver (http://creativecommons.org/publicdomain/zero/1.0/) applies to the data made available in this article, unless otherwise stated. 
From transcriptional and chromatin-based mechanisms, to alternative splicing and post-translational regulation, numerous regulatory levels participate to the control of the floral transition $[9,11-15]$ and its main actors, which have been gathered in the Flowering-Interactive Database (FLOR-ID) [16]. Besides protein regulators involved in developmental transitions, an increasing number of studies have highlighted the regulatory functions of long non-coding RNAs (lncRNAs) [17, 18]. In response to vernalization, the IncRNAs COLDAIR, COLDWRAP, COOLAIR, and Antisense Long participate to the fine regulation of the key MADS-box floral repressor FLOWERING LOCUS C (FLC) via modifications of FLC chromatin environment [19-23]. Recently, FLORE, a Natural Antisense Transcript (NAT) of CYCLING DOF FACTOR5 $(C D F 5)$ was shown to positively regulate flowering time, repressing $C D F$ TFs (CDF1, CDF3, CDF5), and subsequently increasing $F T$ expression [24]. LncRNAs are versatile regulators involved in transcriptional gene regulation, in guiding or scaffolding protein complexes involved in chromatin organization and gene regulation, or even in post-transcriptional regulatory mechanisms [25]. Due to their large number estimated at several thousands and their diversity (intergenic ncRNAs, intronic ncRNAs, antisense RNAs, cis or trans NATs...) [19, 23, 26, 27], their functional annotations and roles in developmental phase transitions remain poorly explored.

The transition to flowering is an integrated process at the scale of the whole plant. Few studies analyzed the transcriptional behaviors of meristematic and root tissues during the floral transition at the genome level [28-32]. Early studies identified few CO targets differentially expressed during flowering in leaves, among which FT [33] which was identified as the major CO target involved in the SD to LD shift response [34]. $F T$ is referred as a flowering integrator with TWIN SISTER OF FT (TSF) [35, 36]. Subsequent studies increased the number of associated genes involved in the leaf response during flowering (see FLOR-ID overview and references therein). However, the dynamics of genome-wide transcriptomes in leaves during the floral transition has not been reported despite the key functions of leaves as receptors of the inductive photoperiodic signal and producer of florigenic molecules. Here, by exploiting the inductive response to a long-day (LD) shift [7] and disconnecting leaf growth or developmental responses from the floral inductive response, we performed a large transcriptome analysis, and identified novel loci and regulatory elements involved in flowering in mature leaves. The transcriptome dataset enabled us to highlight molecular events, providing new insights into transcriptional reprogramming in leaves accompanying the floral transition. Observations of endoreduplication events supported transcriptome data and suggested a novel function in flowering.

\section{Results}

Flowering and organ growth in mature leaves

Our experimental system was based on a photoperiodic shift from SD to LD, which induces a synchronized flowering appropriate to analyze the floral transition (Fig. 1a, Additional file 1: Figure S1a). The floral transition window was determined by the onset of the expression of $C O$ and $F T$, two early markers for flowering and of $A P E$ TALA 1 (AP1), an early marker of the floral meristem identity (Fig. 1b, c). $C O$ and $F T$ expression rapidly increased after the transfer in LD, and reached a maximum at 3 days after transfer (dat), with a slight delay for $F T$ consistently with the primary activating role of $C O$ (Fig. 1b). Although AP1 mRNA transcripts were detected at 7 dat, $A P 1$ expression was slightly earlier (at 5 dat) in meristematic cells when using an AP1::GUS transgenic line, suggesting a completion of the floral transition at that time (Fig. 1b, c, Additional file 1: Figure S1b).

To complete our characterization, we monitored organ growth accompanying the photoperiodic shift. No significant difference was detected in the rosette size during the window of $0-5$ dat between the continuous SD and SD-LD conditions, whereas the first six rosette leaves presented different behaviors (Fig. 1d, Additional file 2: Figure S2). The size of the first two leaves was already established 2 days before transfer (dbt), independently of the photoperiodic conditions. Leaves 3-4 showed no significant differences in growth rate between SD and SD-LD conditions from 0 to 5 dat. Leaves 5-6 presented a higher and continuous growth rate over the 15 days. Thus, during the 5-day floral transition window, developmental and growth processes are arrested in leaves 34 , which makes this pair of mature leaves appropriate material for investigating the early molecular events associated with floral transition, independent of other developmental or signaling events.

\section{Ploidy level changes during the floral transition window}

Since plant development is accompanied by endoreduplication, we analyzed the ploidy distribution in leaves $1-4$. Independently of the leaf position, age and growth conditions, the percentage of $2 \mathrm{C}$ ploidy nuclei was relatively constant (Fig. 2), suggesting a low cell division rate in agreement with the growth analysis. The ploidy levels evolved over time from $4 \mathrm{C}$ to $32 \mathrm{C}$. The dynamics was dependent on the leaf position and the growth conditions. The ploidy levels in leaves $3-4$ in both conditions suggested that endoreduplication events occurred (8C and $16 \mathrm{C}$ nuclei) in response to the LD shift. A population of $32 \mathrm{C}$ nuclei was detected at 15 dat and was 


\section{a}

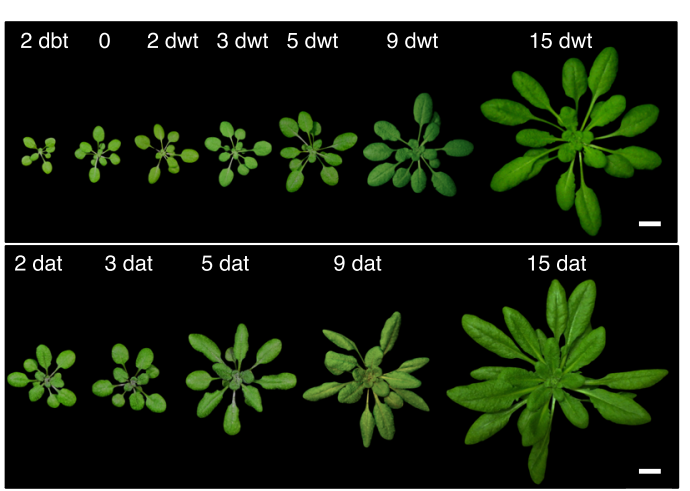

d

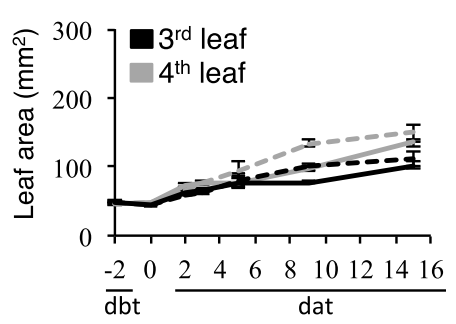

b

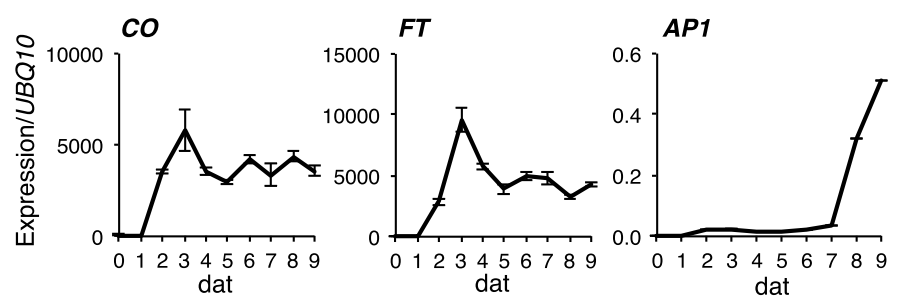

C

AP1::GUS

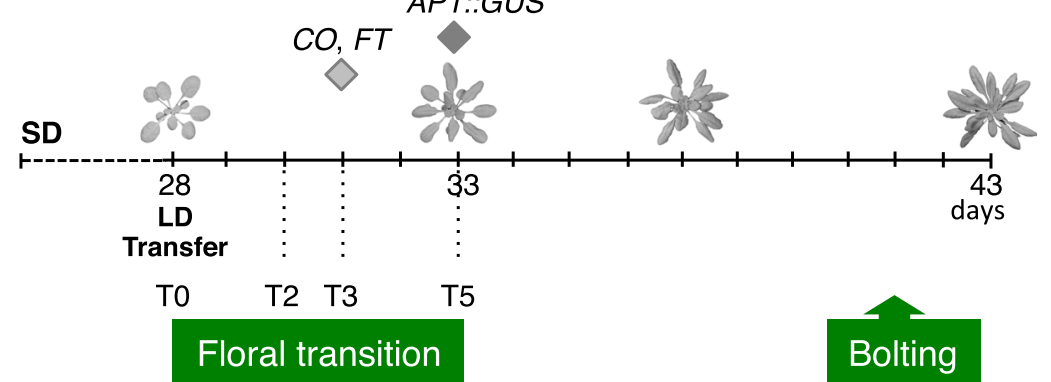

Fig. 1 Floral transition induced by a SD-LD switch in A. thaliana. a Col-0 plants were grown in SD for 4 weeks, kept in SD (upper row) or transferred in LD (bottom row) conditions. Day before transfer (dbt), day without transfer (dwt), day after transfer (dat). Scale bars, $1 \mathrm{~cm}$. b Expression of key flowering time genes. Experimental values are mean \pm SEM. c Schema with developmental events. The CO and FT expression peaks are indicated, whereas the AP1 expression initiation was considered as the upper limit of the floral transition (diamonds). T0, day of transfer in LD. $\mathbf{d}$ Area measurement of the third and fourth rosette leaves. SD conditions (continuous line), transfer in LD (dash line). Experimental values are mean \pm SEM

significantly larger in SD-LD compared to SD conditions. The endoreduplication index evolved from $1.95 \pm$ 0.01 (15 dwt) to $2.35 \pm 0.03$ (15 dat) in leaves 3-4, whereas it was rather constant in leaves $1-2(2.08 \pm 0.02$ at $15 \mathrm{dwt}$ versus $2.04 \pm 0.09$ at $15 \mathrm{dat})$. Thus, the photoperiodic SD-LD switch showed a significant increase in endoreduplication in mature leaves, which occurred during the 3-5 dat window of the floral transition, earlier compared to SD (15 dat).

\section{Major changes in transcription profiles during the inductive shift}

To characterize the molecular events during the floral transition, we examined RNA profiles in leaves 3-4 at different time points (T0, T2, T3, and T5) (Fig. 1c, Additional file 3: Figure S3). We identified 20,284 genes expressed at least in one of the four time points, with more than 6000 differentially expressed genes (DEGs) at the largest transition (T0/T2) and specific gene sets at the main T0/T2, T2/T3 and T3/T5 transitions. By assembling a non-redundant dataset of 14,621 long non-coding transcription units (lncTUs) based on TAIR annotations and published datasets, we also identified 531 differentially expressed lncTUs (DE-lncTUs) (Additional file 4: Figure S4, Additional file 5). These data endorse the highly dynamic transcriptional activity in mature leaves in response to the SD-LD switch.

To characterize the transcriptome profiles, we performed a clustering analysis using different clustering methods and transformation functions ( $R$ package coseq). Twenty-four clusters formed by genes and lncTUs (Additional file 6) were identified and further 
a
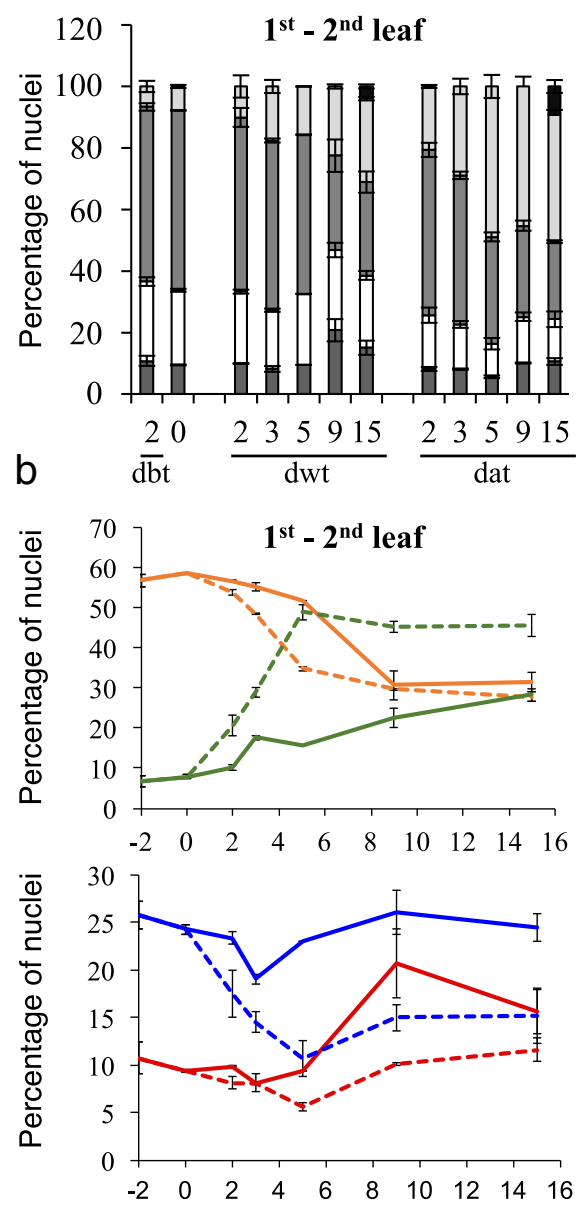

$\overline{\mathrm{dbt}}$
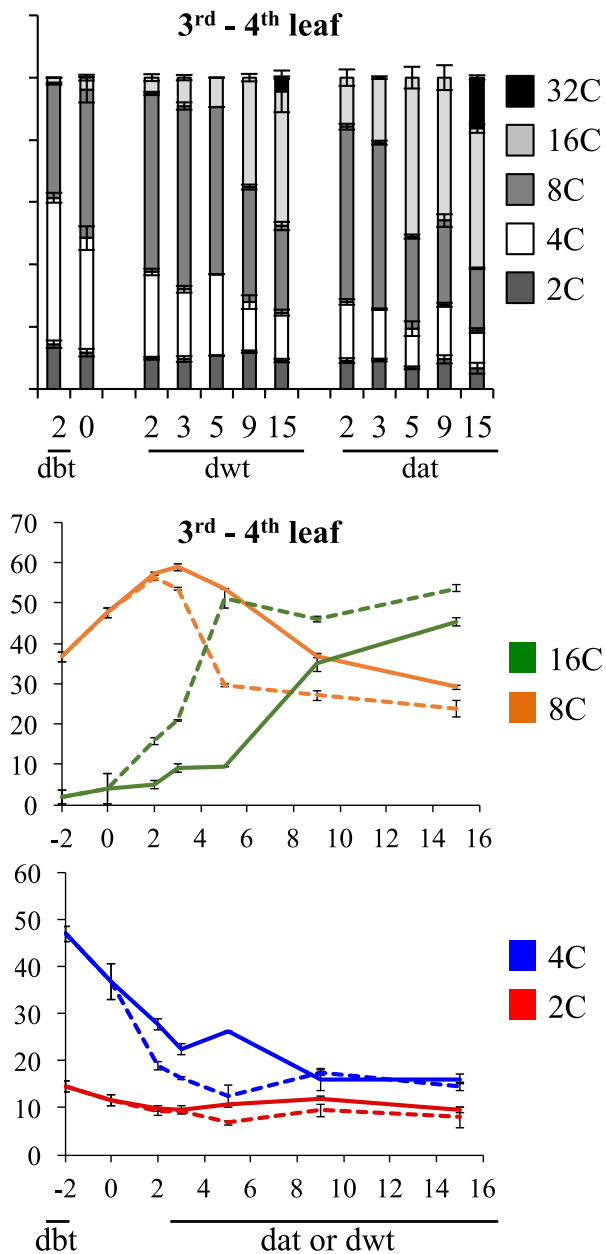

Fig. 2 Ploidy dynamics in leaves during the floral transition. Plants were grown 4 weeks in SD, then either, kept in SD or transferred in LD for 15 days. a Cumulative distributions. $32 \mathrm{C}$ nuclei were only detected at 15 dat. b Dynamics of the distribution of the $2 \mathrm{C}$ to $16 \mathrm{C}$ nuclei. Experimental values are mean \pm SEM. dbt, day before transfer. Dwt, day without transfer (continuous line). dat, day after transfer (dash line)

grouped into 6 cluster families (CF1 to CF6) according to expression tendencies (Fig. 3a, b): transiently-up (CF1) or down (CF2), stable high up (CF3) or down (CF5), stable low up (CF4) or down (CF6).

The 24 clusters had specific GO term enrichments, even among cluster families, such as seven clusters (C20, C23, C16, C18, C19, C15, C4, C3) with strong signatures, thus supporting the cluster analysis (Fig. 3c, d, Additional file 7). This classification revealed different processes in mature leaves during the SD-LD response. For instance, C3, C15, C17 and C24 were enriched in down-regulated genes involved in photosynthesis chlorophyll biosynthesis process, light harvesting, or plastid organization, pointing at a reprogramming of the photosynthetic apparatus. C12 and C15 from CF5 and most clusters in CF3 were enriched in stress-associated terms, such as defence response, response to stimuli, response-to-wounding, highlighting a stress association with the changes in photoperiod, light and perturbation of the circadian clock. In accordance, we noticed in C19, $\mathrm{C} 2$ and C14, "child" GO terms associated with jasmonic acid, salicylic acid or brassinosteroids, respectively. Secondary metabolites, such as flavonoids (C18) and the defence-related glucosinolates (C4), and carbon metabolism (GO terms such as "glucan catabolic process", "cellular polysaccharide catabolic process" in C17) were also modified. We observed enrichments in GO terms associated with cell wall, such as "cell wall organization" and "xyloglucan metabolic process" in C14 or "plant-type secondary cell wall biogenesis" and "cellulose metabolic process" in C20. For instance, XYLOGLUCAN ENDOTRANSGLUCOSYLASE/HYDROLASE 9 (XTH9) involved in cell wall loosening was up-regulated in C14 $(\log 2$ ratio 1.85 , FDR $1.28 \mathrm{E}-3$ at T0/T2). These data suggest that the SD-LD switch is accompanied by cell wall remodeling and some cell wall plasticity in the mature 


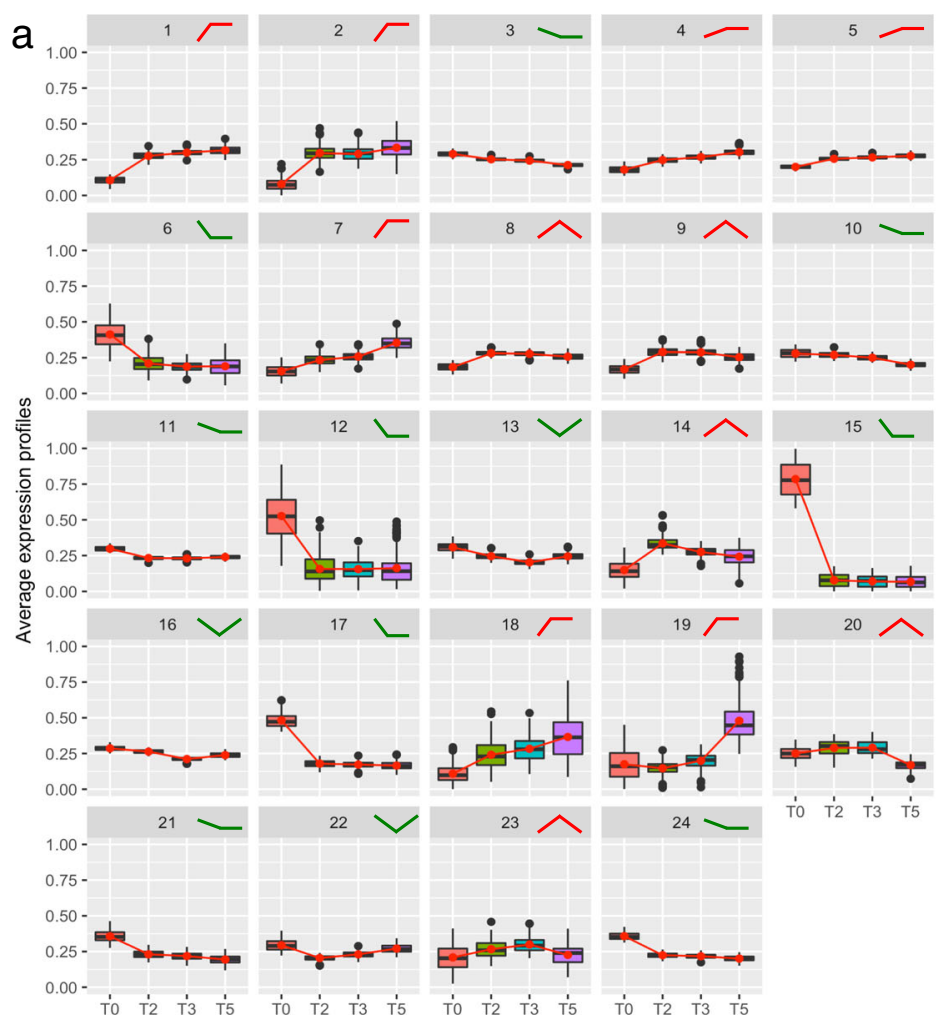

b Family Description Cluster Genes IncTUs

\begin{tabular}{|c|c|c|c|c|c|}
\hline CF1 & Dynamic & $\mathrm{COB}$ & 769 & 7 & 0.9 \\
\hline$\widehat{\gamma}$ & & $\begin{array}{l}\mathrm{C} 09 \\
\mathrm{C} 14 \\
\mathrm{C} 20 \\
\mathrm{C} 23\end{array}$ & $\begin{array}{l}569 \\
160 \\
284 \\
104\end{array}$ & $\begin{array}{l}34 \\
4 \\
23 \\
5\end{array}$ & $\begin{array}{l}5.6 \\
2.4 \\
7.5 \\
4.6\end{array}$ \\
\hline CF2 & $\begin{array}{l}\text { Dynamic } \\
\text { down }\end{array}$ & $\mathrm{C} 13$ & 536 & 22 & 3.9 \\
\hline 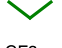 & & $\begin{array}{l}\mathrm{C} 16 \\
\mathrm{C} 22\end{array}$ & $\begin{array}{l}502 \\
323\end{array}$ & $\begin{array}{l}10 \\
19\end{array}$ & $\begin{array}{l}2.0 \\
5.6\end{array}$ \\
\hline CF3 & Up high & $\begin{array}{l}\mathrm{C} 01 \\
\mathrm{C} 02 \\
\mathrm{C} 07 \\
\mathrm{C} 18\end{array}$ & $\begin{array}{l}425 \\
367 \\
580 \\
98 \\
\end{array}$ & $\begin{array}{l}13 \\
32 \\
58 \\
104\end{array}$ & $\begin{array}{l}3.0 \\
8.0 \\
9.1 \\
51.5\end{array}$ \\
\hline CF4 & Up low & $\begin{array}{l}199 \\
\text { C04 }\end{array}$ & $\begin{array}{l}844 \\
841\end{array}$ & 20 & $\begin{array}{l}3.7 \\
2.3\end{array}$ \\
\hline CF5 & Down high & $\begin{array}{l}\text { C06 } \\
\text { C12 } \\
\text { C15 }\end{array}$ & $\begin{array}{l}394 \\
361 \\
98 \\
162\end{array}$ & $\begin{array}{l}10 \\
45 \\
60 \\
11\end{array}$ & $\begin{array}{l}1.0 \\
11.1 \\
38.0 \\
6.4\end{array}$ \\
\hline CF6 & Down low & $\begin{array}{l}\mathrm{C} 03 \\
\mathrm{C} 10 \\
\mathrm{C} 11 \\
\mathrm{C} 21 \\
\mathrm{C} 24\end{array}$ & $\begin{array}{l}530 \\
410 \\
699 \\
528 \\
607\end{array}$ & $\begin{array}{l}7 \\
11 \\
9 \\
38 \\
13\end{array}$ & $\begin{array}{l}1.3 \\
2.6 \\
1.3 \\
6.7 \\
2.1\end{array}$ \\
\hline
\end{tabular}

C
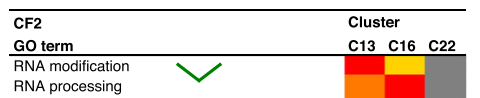

RNA processing

ncRNA processing

cellular nitrogen compound metabolic process

nitrogen compound metabolic process

rRNA processing

ribonucleoprotein complex biogenesis

ribosome biogenesis

translation

peptide biosynthetic process

amide biosynthetic process

peptide metabolic process

cellular amide metabolic process

organonitrogen compound biosynthetic process

organonitrogen compound metabolic process

gene expression

celluar iltrogen compound biosynthetic process

celluar macromolecule biosynthetic process

cellular protein metabolic procs

cellular macromolecule metabolic process

cellular component biogenesis

macromolecule metabolic process

protein metabolic process

cellular biosynthetic process

organic substance biosynthetic process

biosynthetic process

primary metabolic process

cellular metabolic process

organic substance metabolic proces

component organization or biogenesis

cellular process

ribosomal large subunit biogenesis

ribosome assembly

ribonucleoprotein complex assembly

ribonucleoprotein complex subunit organization

ribosomal large subunit assembly

organelle assembly

cellular macromolecular complex assembly

ribosomal small subunit biogenesis

translational elongation

cellular response to hormone stimulus
cellular response to endogenous stimulus

cellular response to organic substance

macromolecular complex assemb

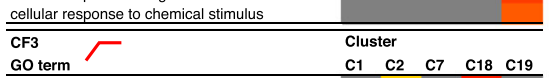

GO term

flavonoid metabolic process

response to stimulus

response to wounding

response to oxygen-containing compound

response to acid chemical

response to chemic

response to stress

response to endogenous stimulus

response to biotic stimulus

response to other organism

response to external biotic stimulus

response to jasmonic acid

response to organic substance

response to hormone

multi-organism process

response to abiotic stimulus

response to inorganic substance

response to fungus

CF4

$\begin{array}{lllll}\text { C1 } & \text { C2 } & \text { C7 } & \text { C18 } & \text { C19 }\end{array}$

CF5

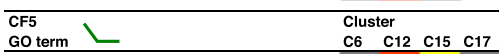

GO term

response to stress
response to stimulus

photosynthesis, light harvesting in photosystem I

response to abiotic stimulus

protein-chromophore linkage

photosynthesis, light harvesting

response to light stimulus

response to radiation

response to temperature stimulus

response to cold

response to red or far red ligh

photosynthesis

response to low light intensity stimulus

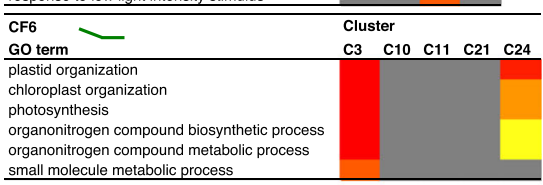

glucosinolate biosynthetic proces

small molecule metabolic process

Fig. 3 Hierarchical clustering of DEGs and DE-IncTUs using their expression levels. a Expression profiles of the 24 clusters. b Composition of the clusters and their organization into 6 cluster families (CFs) according to their general expression tendencies. c-d Heat maps with the biological process GO terms of the clusters per family using SEACOMPARE. Only GO terms with FDR $<10-8$ at least in one of the clusters are presented 
leaves in response to environmental changes. Cell wall modifications were reported in roots in response to inorganic phosphate starvation or in hypocotyl in response to light signaling [37]. Such examples of cell wall remodeling in relation to environment signaling remain rarely reported, especially in leaf. We also noticed in C14 a GO term "response to cyclopentenones", which are fatty acid derivatives with signaling activities. C16 cluster, belonging to CF2 with a transient down-regulation profile, had the highest enrichment terms related to translation, RNA processing and metabolism, suggesting that a strong modification of the protein metabolism at the cellular level is possibly escorting the transition of the metabolic regime occurring in the whole plant during the switch to reproductive phase. Such transient modifications of translation and associated processes were also observed during a cell dedifferentiation and re-differentiation process in Arabidopsis protoplasts [38]. Finally, C20 was enriched in GO terms associated with cell cycle processes. The modifications of the expression of LGO/SMR1, KRP2 and KRP6 cell cycle inhibitor genes involved in endoreduplication [39], the $C Y C A 2 ; 3$, a suppressor of endocycles, the major cell-cycle markers, CDKB2.1, CYCA1;1, and WEE1, a negative regulator of the entrance in the $M$ phase $[40,41]$ were consistent with the onset of the observed endocycles (Additional file 8: Figure S5). In summary, leaf transcriptome during the floral transition revealed major changes in numerous processes, such as carbon and secondary metabolism, signaling events, and endoreduplication.

\section{Flowering and hormone-related genes are differentially expressed in mature leaves during the floral induction} The FLOR-ID core database records genes (FLGs) related to the different regulatory pathways of flowering time and to flower development [16]. To better characterize the molecular events, we analyzed the expression of these genes in mature leaves during the floral transition. We identified a set of 173 DE-FLGs out of the 413 FLGs. These genes were rather evenly distributed among different clusters and regulatory pathways (Fig. 4a, b, Additional file 9). As expected, the major floral integrators were upregulated, such as FT, TWIN SISTER OF FT (TSF), BROTHER OF FT (BFT), CO and COL1. The 173-gene set also included flower meristem identity and flower development (FMI-FD) genes, such as AP2, PETAL LOSS and SEPALLATA 4. Consistently with the experimental design based on a photoperiod shift, most of the FLOR-ID genes classified as associated with the circadian clock were differentially expressed (84.2\%). This large number of DEGs from FLOR-ID in mature leaves highlighted how complex and broad the regulatory gene network of the floral transition is.

In the Arabidopsis Hormone Database (AHD) [42], we identified 331 DEGs involved in hormonal regulation from biosynthesis, metabolism, perception, and transport to hormonal responses. C23 was the most enriched cluster with these genes (Additional file 10; Additional file 11: Figure S6). We noticed that genes related to abscisic acid (ABA) and auxin were the most represented ones among the DEGs (Fig. 4c). Genes involved in the ABA biosynthesis were down-regulated in agreement with a repressive role of $\mathrm{ABA}$ in flowering [43]. However, the switch also largely impacted genes related to hormone signal transduction such as auxin transport (Fig. 4d, e). Among the DEGs involved in auxin-hormone transport, most genes were up-regulated at least transiently during the switch, such as several PIN members (Fig. 4f), questioning the role of auxin transporters in the flowering time control. Genes involved in gibberellin (GA) biosynthesis were mainly down-regulated, while GAMT2, a methyltransferase involved in the GA metabolism was activated, as well as negative regulators of GA responses (RGA-LIKE1-2) (Additional file 11: Figure S6b). Indeed, genes involved in cytokinin (CK) biosynthesis and the SOB FIVE-LIKE 1, 2 genes (SOFL1, 2), which participate to $C K$ level regulation [44] were activated, whereas genes involved in CK catabolism (CKK4, CKK6) were down-regulated. Consistently, the type-B ARABIDOPSIS RESPONSE REGULATOR 10 (ARR10) TF, a key player in the CK signaling pathway for the light response and shoot initiation [45], was up-regulated between T0/T5. Beside DEGs involved in one hormonal pathway, 36 DEGs are involved in hormonal crosstalk, with ABA being involved in most of these crosstalk (Additional file 12). Whereas GA is proposed to promote the floral transition and have antagonistic effects with ABA, our data suggest a complex hormonal interplay during the SD-LD switch, with GA, ABA but also new players such as the brassinosteroids and derivative forms, as well as IAA and CKs.

\section{Novel regulatory actors involved in the SD-LD switch}

To identify regulatory elements associated with flowering in mature leaves, we performed an $a b$ initio search of motifs amongst the promoters of coregulated genes in each cluster, using the Preferentially Located Motifs (PLM) detector algorithm [46]. We identified 192 significant motifs of 4 to 11 bases, distributed over the 24 clusters (M001 to M192, $p$-value <0.05) (Fig. 5, Additional file 13). We then restricted our analysis to the seven-mers or longer motifs (91 motifs). Their occurrence varied from a unique motif (M055) in 11 different clusters to 20 motifs present in only one cluster. We questioned whether these motifs corresponded to previously discovered transcription factors bindings sites (TFBSs) by using the Tomtom Motif Comparison Tool [47] and two databases of functional motifs identified by protein-binding microarrays (PBMdb) [48], and by DAP-seq (DAPdb) [49]. We kept only significant motifs 


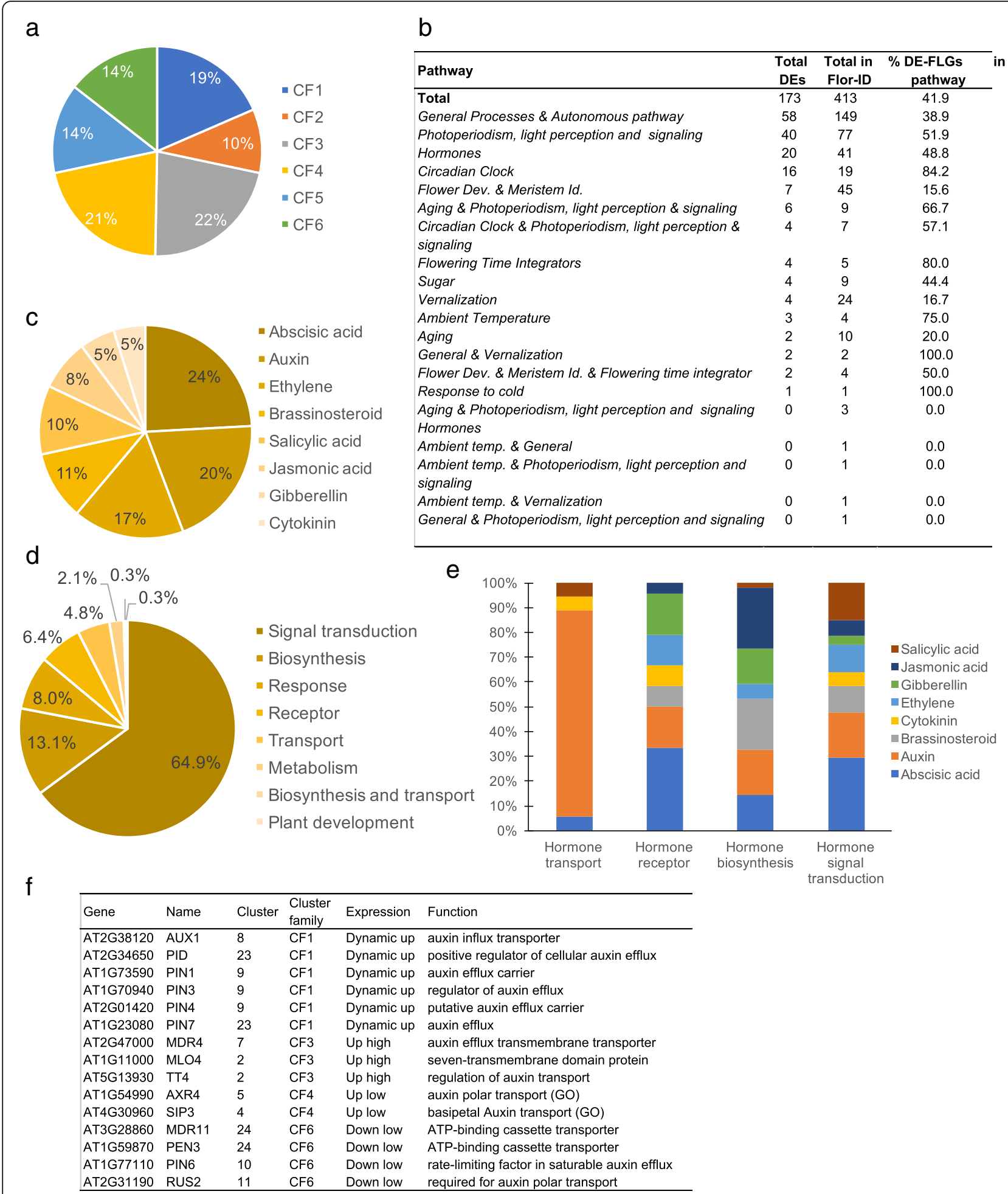

Fig. 4 Analyses of genes differentially expressed during the SD-LD switch involved in flowering (FLGS) and hormone responses. a Distribution of the DE-FLGs in the different cluster families. $\mathbf{b}$ Repartition of the DEGs in the regulatory pathways involved in flowering. $\mathbf{c}, \mathbf{d}$ Distribution of DEGs belonging to the AHD according to hormones (c) and to the pathways (d). e Distributions in the 5 main functional pathways. $\mathbf{f}$ DEGs related to auxin transport 


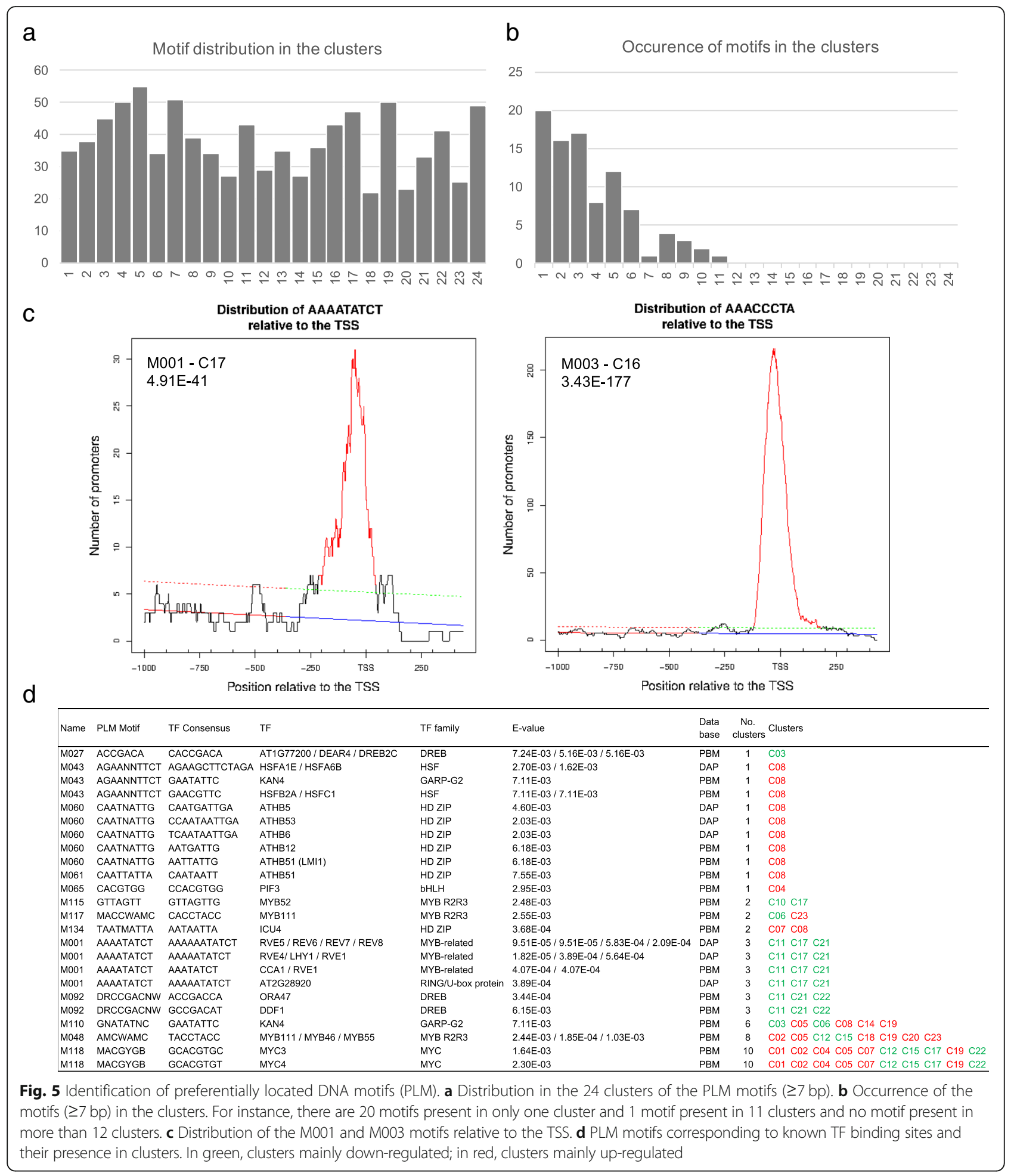

(E-value <0.01; for DAP motifs, overlap value larger than $75 \%$ ). Thirteen motifs were similar to known motifs targeted by 32 TFs from these databases (Fig. 5), the remaining motifs correspond to putative novel regulatory elements, whose function will require further investigation.
The 32 TF set was enriched in GO terms associated with "regulation of metabolic process" (GO:0019222; $p$-value 5.65E-29, FDR 1.11E-26), "response to hormone stimulus" (GO:0009725; $p$-value 3.86E-12, FDR 3.53E-10) and "circadian rhythm" (GO:0007623; $p$-value 8.68E-10, FDR 6.43E-08) (PlantGSEA toolkit). The M001 motif 
(AAAATATCT) matched to the TFBS recognized by CCA1, LHY1, RVE1, and RVE5-8 TFs and to the "Evening Element", involved in the control of circadian-regulated genes [50] and identified, for instance, in down-regulated genes such as SVP, PHYTOCLOCK 1 (PCL1) and LONG VEGETATIVE PHASE 1 (LOV1). M118 (MACGYGB) is similar to the TFBS of the MYC3 and MYC4, two TFs involved in flowering [51]. Intriguingly, no TF could be associated with M003 (AAAC CCTA) and M004 (AAACCCTAA), the two closely related motifs with the best PLM $p$-values (3.43E-177, 5.39E-104, respectively). Remarkably, M003 was highly similar to the (A/G/T)AACCCTA(A/G) motif, an LHP1 binding motif, related to the telo-box motif (AAACCCTA) and recognized by REPEAT BINDING PROTEIN1 (TRB1) [52], and to a lesser extent, to the tertiary motif of TOE1 (AACCTTAA), a TF belonging to the AP2/EREBP superfamily (E-value 0.54 using the PBMdb). Both LHP1 and TOE1 are known to repress flowering, LHP1 being a component of PRC1 complex $[53,54]$ and TOE1 inhibiting the $\mathrm{CO}$ activity in the $F T$ activation [55]. A majority of these 32 TFs (56\%) were differentially regulated during the process, implying functional preferences of the identified motifs.

We thus further analyzed the expression of TFs by using the PlantTFDB [56], and identified 648 differentially expressed TFs, belonging to $51 \mathrm{TF}$ families (Additional file 14). FT, TSF and BFT were co-regulated with 7 other TFs (among which, NAC3, NLP3, WOX2, and ASG4) (C18) and CO and COL1 with 38 other TFs (C02), enlarging regulatory networks. The transiently-up C23 cluster from CF1 had the highest percentage of TFs among its DEGs, in agreement with the largest transcriptional switch occurring at T0/T2 (Fig. 6a). For some TF families, a large proportion of the members were differentially expressed, suggesting important roles of these families in the response to the SD-LD shift. One of these family, the family of BBX proteins, comprises the DBB (double B-box (BBX) zinc finger protein subfamily and the CO-like subfamily $[57,58]$ (mainly down-regulated; 10 out of the 17 DEGs) (Fig. 6b). The BBX family comprises regulators involved in the circadian clock, photomorphogenesis, flowering time, flower development, or stress responses, such as the flowering activator COL5 [59], BBX2/COL1 a circadian clock regulator [57] or the flowering repressor COL9 [60]. These data indicate putative synergistic and antagonistic roles among the BXX family for the SD-LD switch. Thirty-nine other TFs in C2, among which 5 unknown TFs may be putative novel candidates for flowering time control in the mature leaf. The expression of the NF-Y (Nuclear Factor Y) family was also largely altered. The conserved NF-Y complexes are composed of NF-YA, NF-YB and NF-YC subunits and involved in development, stress response and flowering [61-64]. Here, the NF-YA subunits were mainly up-regulated (8 out 9 NF-YA in CF3 and CF4, the up-high and up-low cluster families) compared to the other subunits (2 NF-YB, 4 NF-YC), and some subunits were coregulated (such as NF-YA4/NF-YB2/ NF-YC2 in CF3, or NF-YA8/NF-YA9/NF-YB3 in CF4), suggesting specific and dynamic compositions of NF-Y complexes. The three members of the small WHIRLY (WHY) family of single-stranded DNA binding proteins were also mainly down-regulated (C10, C13). WHIRLY1 was proposed to be involved in the gene regulation and chloroplast-to-nucleus retrograde signaling in response to redox processes occurring during light adaptation $[65$, 66]. While WHIRLY1 has a dual chloroplastic/nuclear localization, WHY2 and WHY3 are targeted to mitochondria and chloroplast, respectively. These data suggest a novel chloroplast-to-nucleus signaling in flowering time control.

Among FLOR-ID, a functionally related subset of 64 TFs, corresponding to the differentially expressed TFs of the database (64 out of 143) (Additional file 15) was analyzed using the TF2Network tool [67] to decipher gene regulatory networks in the mature leaf. We identified 66 specific candidate regulators, by comparison with the subset corresponding to the FLOR-ID TFs, which were not differentially expressed (data not shown). The best-ranked regulators were $\mathrm{HYH}, \mathrm{ABF} 1, \mathrm{TCP} 21, \mathrm{ABI}$, MYC2 and HY5 (Additional file 16: Figure S7), suggesting candidate regulators of the floral transition in mature leaf. The identification of ABI5 and ABF1 as candidate regulators, which are TFs involved in ABA responses, was in agreement with the high percentage of ABA-related genes differentially expressed in mature leaf during the floral transition (Fig. 4c).

Finally, since chromatin is a key transcriptional regulatory level, we searched for key chromatin-associated genes (CAGs) involved in flowering. We identified 90 DE-CAGs, 91\% being differentially expressed at T0/T2, with a bias towards up-regulated genes (Additional files 17 and 18). We noticed that a large proportion (39\%) of the DE-CAGs encoded histone variants with, for instance, the H1.3 variant (HON3), associated with stress response, $5 H 3.1$ variants, which are incorporated in a replication-dependent manner in agreement with the endoreduplication events or $10 \mathrm{H} 2 \mathrm{~A}$ variants (Fig. 6c). Among the genes involved in histone post-translational modifications, such as genes encoding SDG4/ASH1-RELATED 3 and SDG13/SUVR1 histone methyltransferases, were up-regulated, whereas histone deacetylases were only weakly differentially expressed, except HDA2, which was down-regulated and associated with the floral transition for the first time here. Consistently with modifications in DNA methylation accompanying early floral transition events, we observed the expression changes of 
a

\begin{tabular}{llll}
\hline Cluster & \multicolumn{2}{l}{ No. Genes } & No. TFs $\%$ TFs \\
\hline C23 & 104 & 16 & 15.4 \\
C15 & 162 & 21 & 13.0 \\
C06 & 361 & 43 & 11.9 \\
C01 & 425 & 47 & 11.1 \\
C02 & 367 & 40 & 10.9 \\
C07 & 580 & 52 & 9.0 \\
C14 & 160 & 14 & 8.8 \\
C19 & 184 & 16 & 8.7 \\
C09 & 569 & 47 & 8.3 \\
C04 & 841 & 64 & 7.6 \\
C18 & 98 & 7 & 7.1 \\
C22 & 323 & 23 & 7.1 \\
C21 & 528 & 37 & 7.0 \\
C05 & 994 & 66 & 6.6 \\
C08 & 769 & 46 & 6.0 \\
C17 & 306 & 18 & 5.9 \\
C12 & 98 & 5 & 5.1 \\
C11 & 699 & 25 & 3.6 \\
C24 & 607 & 21 & 3.5 \\
C20 & 284 & 9 & 3.2 \\
C10 & 410 & 9 & 2.2 \\
C13 & 536 & 10 & 1.9 \\
C03 & 530 & 8 & 1.5 \\
C16 & 502 & 4 & 0.8 \\
\hline & & &
\end{tabular}

C

\begin{tabular}{|c|c|c|c|c|c|c|}
\hline Gene ID & Symbol & & Family & $\begin{array}{c}\text { T0/T2 } \\
\text { log2Fold } \\
\text { Change }\end{array}$ & $\begin{array}{c}\text { T2/T3 } \\
\text { log2Fold } \\
\text { Change }\end{array}$ & $\begin{array}{c}\text { T3/T5 } \\
\text { log2Fold } \\
\text { Change }\end{array}$ \\
\hline AT2G1805 & HON3 & $\mathrm{H} 1.3$ & histone $\mathrm{H} 1$ & 1.01 & & 0.78 \\
\hline AT5G5987 & HTA6 & H2A.W & histone & 1.12 & & -0.49 \\
\hline AT2G3881 & HTA8 & H2A.Z & histone & 1.03 & & -0.31 \\
\hline AT3G5456 & HTA11 & & histone & 0.91 & & -0.23 \\
\hline AT1G5106 & HTA10 & & histone & 0.64 & & -0.44 \\
\hline AT3G2067 & HTA13 & & histone & 0.61 & & -0.34 \\
\hline AT5G2767 & HTA7 & H2A.W & histone & 0.58 & & -0.41 \\
\hline AT4G2723 & HTA2 & & histone & 0.53 & & -0.31 \\
\hline AT5G5464 & HTA1 & RAT5 & Histone & 0.52 & & -0.37 \\
\hline AT1G0888 & HTA5 & H2A.X & Histone & 0.32 & & -0.11 \\
\hline AT5G2288 & HTB2 & & histone & 1.37 & & -0.19 \\
\hline AT5G0257 & HTB10 & & histone & 1.01 & & -0.38 \\
\hline AT3G5365 & HTB6 & & histone & 0.93 & & -0.5 \\
\hline AT3G4603 & HTB11 & & histone & 0.43 & & -0.29 \\
\hline AT3G4598 & HTB9 & & histone & 0.34 & & -0.34 \\
\hline AT5G5991 & HTB4 & & histone & 0.33 & & -0.17 \\
\hline AT1G0137 & HTR12 & CENH & Histone $\mathrm{H} 3$ & 0.67 & & -0.3 \\
\hline AT5G6536 & HTR1 & H3.1 & Histone $\mathrm{H} 3$ & 1.07 & & -0.3 \\
\hline AT5G1040 & HTR13 & H3.1 & Histone $\mathrm{H} 3$ & 1.03 & & -0.46 \\
\hline AT1G0920 & HTR2 & H3.1 & Histone $\mathrm{H} 3$ & 0.98 & & -0.39 \\
\hline AT5G1039 & HTR9 & H3.1 & Histone $\mathrm{H} 3$ & 0.96 & & -0.05 \\
\hline AT3G2736 & HTR3 & H3.1 & Histone $\mathrm{H} 3$ & 0.92 & & -0.57 \\
\hline AT5G5969 & HFO2 & & histone $\mathrm{H} 4$ & 1.03 & & -0.3 \\
\hline AT3G4632 & HFO1 & & histone $\mathrm{H} 4$ & 0.85 & & -0.46 \\
\hline AT3G4593 & HFO7 & & histone $\mathrm{H} 4$ & 0.85 & & -0.31 \\
\hline AT5G5997 & HFO6 & & histone $\mathrm{H} 4$ & 0.78 & & -0.43 \\
\hline AT2G2874 & HFO3 & & histone $\mathrm{H} 4$ & 0.72 & & -0.54 \\
\hline AT1G0782 & HFO4 & & histone $\mathrm{H} 4$ & 0.45 & & -0.35 \\
\hline AT3G5373 & HFO5 & & histone $\mathrm{H} 4$ & 0.35 & & -0.32 \\
\hline AT5G0256 & HTA12 & H2A.W & histone & -0.39 & & 0.1 \\
\hline AT2G3062 & HON2 & & histone $\mathrm{H} 1$ & -0.39 & & -0.13 \\
\hline AT5G0878 & HON8 & & histone $\mathrm{H} 1$ & -0.52 & & 0.01 \\
\hline AT2G2872 & HTB3 & & histone & -1.75 & & -0.05 \\
\hline AT1G0779 & HTB1 & & histone & & & -0.4 \\
\hline AT2G3747 & HTB5 & & histone & & & -0.47 \\
\hline
\end{tabular}

b

\begin{tabular}{|c|c|c|c|c|c|}
\hline ID & Gene family & Name & Gene Symbols & $\begin{array}{l}\text { Cluster } \\
\text { ID }\end{array}$ & $\begin{array}{l}\text { Cluster } \\
\text { Family }\end{array}$ \\
\hline AT1G68520 & CO-like & B-BOX DOMAIN PROTEIN 14 & BBX14/COL6 & 8 & CF1 \\
\hline AT4G38960 & DBB & B-BOX DOMAIN PROTEIN 19 & BBX19/DBB1b & 9 & CF1 \\
\hline AT2G31380 & DBB & SALT TOLERANCE HOMOLOGUE & BBX25/STH/STH1 & 9 & CF1 \\
\hline AT3G02380 & Co-like & CONSTANS-LIKE 2 & $\mathrm{BBX} 3 / \mathrm{COL} 2$ & 9 & CF1 \\
\hline AT2G33500 & Co-like & B-BOX DOMAIN PROTEIN 12 & BBX12/COL14 & 13 & CF2 \\
\hline AT1G28050 & Co-like & B-BOX DOMAIN PROTEIN 13 & BBX13/COL15 & 22 & CF2 \\
\hline AT5G15840 & DBB & CONSTANS & $\mathrm{BBX} 1 / \mathrm{CO}$ & 2 & CF3 \\
\hline AT5G15850 & Co-like & CONSTANS-LIKE 1 & BBX2/COL1 & 2 & CF3 \\
\hline AT3G07650 & Co-like & CONSTANS-LIKE 9 & BBX7/COL9 & 1 & CF3 \\
\hline AT2G47890 & DBB & B-BOX DOMAIN PROTEIN 121 & BBX11/COL13 & 15 & CF5 \\
\hline AT1G73870 & Co-like & B-BOX DOMAIN PROTEIN 16 & BBX16/COL7 & 6 & CF5 \\
\hline AT1G49130 & Co-like & B-BOX DOMAIN PROTEIN 17 & BBX17/COL8 & 6 & CF5 \\
\hline AT2G21320 & DBB & B-BOX DOMAIN PROTEIN 18 & BBX18/DBB1a & 6 & CF5 \\
\hline AT1G78600 & DBB & LIGHT-REGULATED ZINC FINGER PROTEIN 1 & BBX22/LZF1/STH3/DBB3 & 15 & CF5 \\
\hline AT1G06040 & DBB & SALT TOLERANCE & BBX24/STO & 15 & CF5 \\
\hline AT5G57660 & CO-like & CONSTANS-LIKE 5 & BBX6/COL5 & 15 & CF5 \\
\hline AT5G48250 & CO-like & B-BOX DOMAIN PROTEIN 8 & BBX8/COL10 & 24 & CF6 \\
\hline
\end{tabular}

Fig. 6 TFs and chromatin-associated genes differentially expressed during the floral transition. a TF Distribution in the clusters. $\mathbf{b}$ B-Box containing TFs. c Histone-related genes 
MET1, CMT3 but also of DEMETER-LIKE2 (DML2), encoding a DNA glycosylase involved in active DNA demethylation, in the mature leaves. Thus, the results suggest a rapid modification of the epigenome, concomitant with the changed TF profiles, which further endorses a dramatic reprogramming in the leaf genome, reminiscent of another developmental switch [38].

\section{A set of IncTUs is differentially regulated during the floral transition}

To identify lncTUs with putative regulatory function, we firstly questioned whether the 14,621 lncTUs regions of our dataset and the $531 \mathrm{DE}$-lncTUs were associated with specific genome topographical characteristics, especially the nine chromatin states (CS) [68] (Fig. 7a). Consistently, lncTUs were globally preferentially associated with CS4 that corresponds predominantly to distal promoter regions and non-coding intergenic regions. CS4 has high levels of H3K27me3 and reduced levels of active histone marks. To a lesser extent, but still significantly, lncTUs are prevalent in CS5, the Polycomb-regulated CS also enriched in high H3K27me3 levels, and in CS8, the AT-rich heterochromatin, but not with the constitutive heterochromatin (CS9). Whereas the fold changes for lncTUs and DE-lncTUs were quite similar in CS8, a large discrepancy was observed between the two sets in the bivalent chromatin state, CS2. We observed a low number of DE-lncTUs in regions targeted by PCF11-SIMILAR PROTEIN 4 (PCFS4), a key factor involved in flowering time and acting on the FCA alternative processing [69] (Fig. 7a). These results suggest the existence of sets of lncTUs associated with specific chromatin states and with specific regulatory activities during the floral transition, such as the CS2 subset of the 193 DE-lncTUs or the PCSF4 subset.

A hierarchical clustering analysis revealed that the majority of the 531 DE-lncTUs were either up- or down-regulated, whereas only 15 DE-lncTUs changed dynamically (Fig. 7b, c). This indicates that most of the of DE-lncTUs were mainly specific for either the vegetative or the reproductive phase, while fewer specific ones are involved in the transition event only.

To find out if lncTUs can change the expression of genes in cis we examined the expression of both the lncTUs and their neighboring genes. No correlation between expression and structure could be detected at the genome level. We then investigated whether lncTUs may have putative NAT regulatory function by analyzing lncTUs with overlapping genes. The FLORE NAT, for example, represses CDF5 located in antisense but also $C D F 1$ and CDF3 located on other chromosomes and participates to the flowering time control [24]. Whereas $C D F 2$ was up-regulated, $C D F 3$ and $C D F 5$ were down-regulated in our data. Putative lncTUs that we could identified in the regions of $C D F 2, C D F 3$, and CDF5 were not differentially expressed in our experimental design, which suggests that other regulatory mechanisms control $C D F$ expression. Alternatively, transient changes in the expression of the corresponding IncTUs could not be detected. In our dataset, we found that 655 lncTUs overlapped with a flanking gene and were transcribed in antisense (putative NAT lncTUs). Among them, 19 NAT lncTUs showed opposite transcriptional activity with the overlapping gene, in at least one of the 6 comparisons (Fig. 7d, Additional file 19). However, the expression dynamics of the gene and its NAT lncTU may be more complex and the two partners may have no synchronized expression. For instance, a lncTU formed a NAT couple with MAF5 (LNCRNA-MERGE_C-9859, named MAF5_NAT), encoding a floral repressor of the $F L C$ clade. MAF5 was differentially regulated during the floral transition and MAF5_NAT was strongly down-regulated before the up-regulation of MAF5 (Additional file 20: Figure S8). This is different from the transient up-regulation of antisense regulatory ncRNAs which represses FLC, thus illustrating the complexity of the flowering regulation mechanism.

Finally, for each DE-lncTU, we examined the presence of the TFBSs of the SVP, FLC and SOC1 flowering regulators, in a 4-kb window (3 kb upstream - $1 \mathrm{~kb}$ downstream) [70-74]. We identified 123 TFBSs in the vicinity of 63 DE-lncTUs, with one to seven of these binding sites mainly present in the $5^{\prime}$ regions, suggesting some functionality of these TFBSs regarding the expression of the DE-lncTUs and putative roles of these TFs in lncTU regulation (Fig. 7e, f, Additional file 21). Furthermore, some of the DE-lncTUs with TFBSs were located in CS2 and/or involved in NAT couples. Based on these criteria (expression profile, presence of TFBS, location in specific chromatin state, NAT couple), the identified DE-lncTUs represent interesting candidates whose regulatory function in flowering will require further investigation.

\section{Discussion}

Complex molecular processes in mature leaves during the floral transition

In sensing floral inductive stimuli, leaves produce the florigen signal that switches the SAM from the vegetative to reproductive phase. Key genes involved in producing the florigen have been identified, but the global molecular events in leaves during the floral transition remain poorly described. By focusing on mature rosette leaf whose growth was completed, our study completes analyses and highlights molecular events of the floral transition in this organ. Based on differential gene expression profiles, we showed that the floral transition induced by the SD-LD switch is accompanied by 


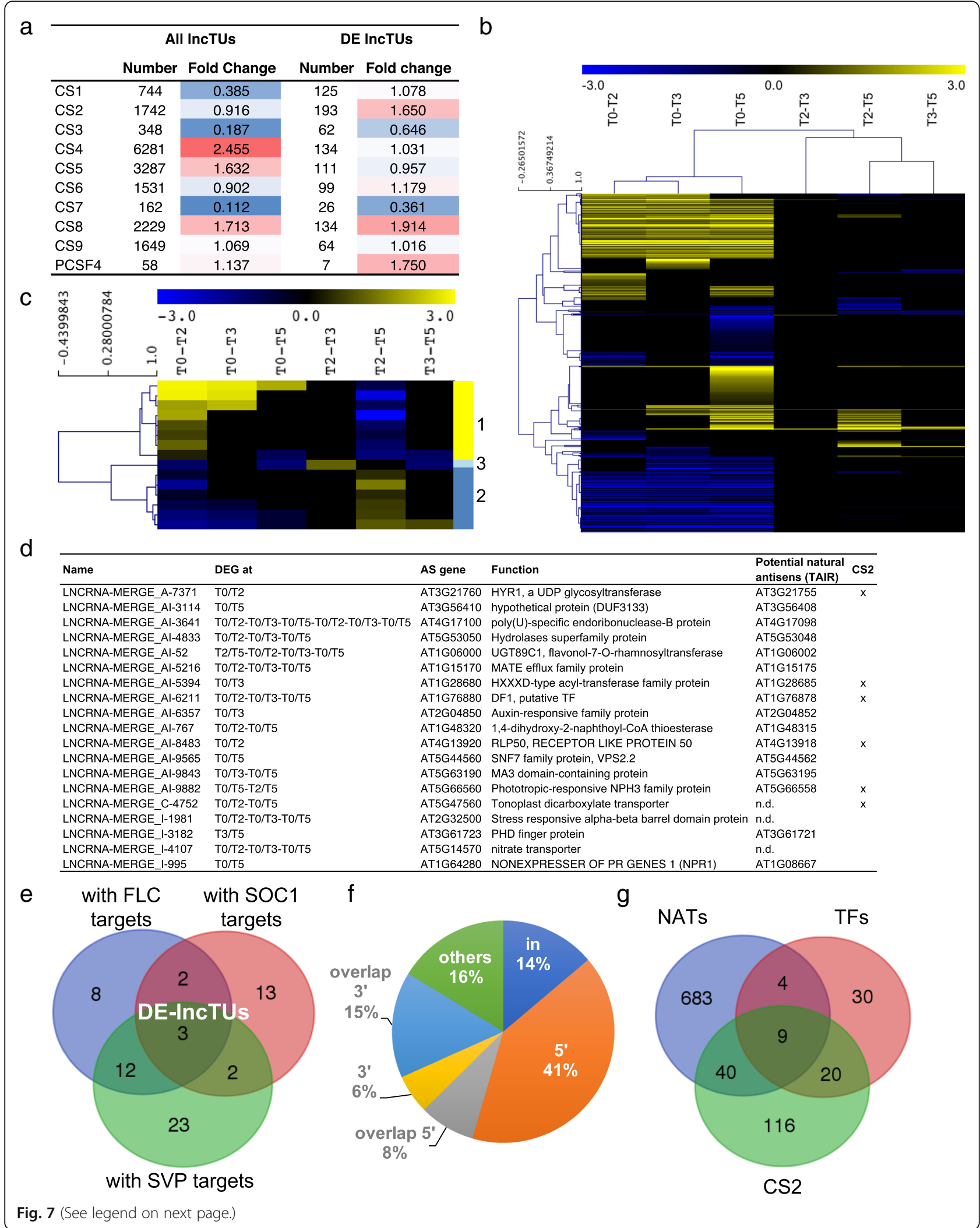


(See figure on previous page.)

Fig. 7 Analyses of IncRNAs during the photoperiodic inductive switch. a Heat maps of the IncTU distribution in the nine chromatin states and the PCFS4 target regions. The fold change was calculated based on the ratio between observed and randomly distributed IncRNAs. $\mathbf{b}$ Hierarchical clustering of the DE-IncTUs. c Hierarchical clustering of the dynamic DE-IncTUs. $\mathbf{d}$ DE-IncTUs in NAT orientation with genes differentially expressed at the same time point and corresponding potential NATs, as annotated in TAIR. e Venn diagram between the different DE-IncTU sets with FLC, SOC1 or SVP binding sites in their vicinities. $\mathbf{f}$ Distribution of the TFBSS of FLC, SOC1 or SVP inside (in), at the 5' or 3' end, overlapping on the 3' or $5^{\prime}$ end of the IncTUs. g Venn diagram between DE-IncTUs in CS2, DE-IncTUs with SVP, FLC or SOC1 binding sites (TFs) in their vicinities and DEIncTUs forming NAT couples

re-organization of photosynthetic capacity, protein synthesis, cellular metabolism, hormonal action, stress response and cell cycle regulation, with an intricate interplay between the light regime, the circadian clock and the floral transition. Our data highlight the complex role of mature leaves in the floral transition.

LD stimulates increases in leaf sucrose level as part of the florigenic signal [75-77]. However, other metabolites are also involved in the floral transition: carbon, phosphorous, nitrogen or sulphur can impact this process [4, 78]. Genes associated with flavonoids and glucosinolates contents were differentially regulated during the photoperiodic switch, whereas these secondary metabolites are usually associated with stress responses [79]. These data support a recent study showing that the flowering regulator $F L C$ is present in a QTL interval associated with glucosinolate contents in the brassicaceae species, Aethionema arabicum [80]. The switch occurring in the SAM appears to require massive metabolic and physiologic reprogramming events in the leaf to further explore.

\section{Highlight of new regulatory candidates in flowering control}

Dynamics changes were reported in the transcriptional profiles of genes but also lncRNAs, highlighting the potential regulatory functions of some of them in the floral transition. Our atlas of lncTUs, with putative regulatory functions based, for instance, on their location in bivalent chromatin states or in antisense with protein-coding genes, provides promising resource for new actors in the genome regulation during the floral transition.

The analysis of the gene clusters allowed the extraction of specific putative regulatory motifs. Some of these DNA elements corresponded to binding sites of differentially expressed TFs, suggesting functionality in the transcriptional regulation of the floral transition. In parallel, a large set of differentially expressed TFs involved in various processes was identified, consistently with the molecular processes highlighted with the GO analysis. Changes in transcription of a set of FMI-FD genes, whose action is crucial in meristems, suggest other levels of gene regulation during the floral transition.

By focusing on FLOR-ID TFs, we highlighted small gene regulatory networks and potentially, new players in the floral transition in leaf, such as ABI5, ABF1, or
TCP21. TCP21 is involved in the circadian clock regulation and controls CCA1 expression [81]. The identification of TCP21 as candidate regulator here is in agreement with the large proportion (84.2\%) of circadian clock-associated genes differentially expressed, during the SD-LD switch (Fig. 4b). ABI5 was reported as a floral repressor [82], which is consistent with its down-regulation here during the SD-LD switch and its putative interactions with the downstream deregulated TFs. ABA is involved in the control of flowering time with opposite effects according to environmental conditions [83-85]. For instance, ABA was shown to promote flowering time in a LD-dependent manner and in response to water resource availability, by modulating GIGANTEA (GI) activity on $F T$ and TSF $[84,85]$. However, the down-regulation of GI here suggests that the ABA-dependent promotion of flowering may not be involved in the SD-LD transition, but a loss of repression mediated by ABI5 may possibly occur. The hormonal contribution of the GA-dependent promoting pathway [10] may also play a role. Most of the hormone pathways being affected during the switch, albeit to different degrees, performing hormone dosages may help to untangle their contribution to flowering time.

\section{Endoreduplication events accompanying the floral transition in leaves}

The acceleration of the cell division was reported in the SAM during the floral transition in A. thaliana $[29,86]$. Here, we report that endocycles escort the floral transition in leaves. Thus, the floral induction is accompanied by modulations of the cell cycle in both leaves and meristems, but with differences in cell cycle exits according to the organs, endocycle or mitosis, respectively. Our result is supported by the changes in expression of key cell cycle phase markers, such as $C Y C A 2 ; 3$, but also histone variants and endocycle-related genes. Consistently with a loss of function stimulating endocycles [87], CYCD3 genes were down-regulated in our experiments. The spindle assembly checkpoint (SAC) genes were also shown to impact both the floral transition and the timing of the endocycle onset [88]. Here, only BUBR1/ MAD3 from the SAC family was slightly down-regulated, but its function in the Arabidopsis mitotic checkpoint control remain poorly documented. 
Hormone signaling pathways participate to the control of the mitotic-to-endocycle transition: the endocycle repression is induced by high auxin contents $[39,89]$. Consistently, we observed alterations of the auxin pathways during the photoperiodic inductive switch. The SUMO E3 ligase HPY2, described as an endocycle repressor, which may link auxin signaling and cell cycle program $[89,90]$, as well as two other negative regulators of endocycles, were up-regulated at the T0/T2 transition, suggesting that the entry into the endocycle program in the mature leaves may result from a fine dosage between the different controlling pathways.

Previous studies showed that the increase in light intensity [91] and UV-B radiation [92] are associated with changes in ploidy levels. A proposed hypothesis is that the ploidy dynamics might be an adaptive response to damage possibly induced by solar radiation. Finally, we could also speculate that the increase in ploidy level in mature leaves during the floral transition may contribute to an increase in energy production required for the developmental switch, an increase in metabolites and endogenous signaling molecules, or a modulation of transcription thresholds.

\section{Conclusions}

Our detailed study provides a novel molecular framework to further question the roles of new putative regulators in leaves during the floral transition, such as new putative lncRNAs, whose polyadenylation status will require further confirmation. Furthermore, it points at the relationship between flowering and endoreduplication and at the complex interplay between several plant hormones, which open new perspectives.

\section{Methods}

\section{Plant materials and growth conditions}

All Arabidopsis thaliana lines were in the Col-0 background. Seeds of the $p A P 1:: G U S$ transgenic line were kindly provided by Prof. Dr. G. Angenent (unpublished material). The AP1 promoter fragment was fused to a GUS-GFP cassette as described in [93], using the binary pBGWFS7 vector from VIB [94]. Plants were grown in growth chamber under SD ( $8 \mathrm{~h}$ light/16 h dark) or LD (16 h light/8 h dark) conditions. White fluorescent light was used. The photosynthetic photon flux density was $120 \mu \mathrm{mol} \mathrm{m}^{-2} \mathrm{~s}^{-1}$ in SD and LD. In SD, the temperature was $21{ }^{\circ} \mathrm{C}$ during the light and $18{ }^{\circ} \mathrm{C}$ during the dark period, and the humidity (65\%) remained constant. In $\mathrm{LD}$, the temperature $\left(21^{\circ} \mathrm{C}\right)$ and the humidity $(70 \%)$ remained constant, $21^{\circ} \mathrm{C}$ and $70 \%$, respectively. Plants were cultured for 3,4 or 5 weeks in soil, in individual pot. The transfer was done at the end of SD light, preceding the LD dark period. Flowering time indicators were recorded as previously described [53](Additional file 1: Figure S1a). The percentages of cauline leaf relatively to the total leaf number (CL\%) quantifies the relationship between bolting and floral transition events [95]. For plants grown 3, 4 and 5 weeks in SD and transferred in LD it similar to the CL\% in continuous LD (17.6\%), and higher compared to continuous SD (11.9\%) [96]. This preliminary assay showed that the SD-LD switch mimicked LD growth conditions. For a good compromise between time and material quantity, analyses were then pursued on plants grown for 4 weeks in SD.

For RNA extraction, plants were collected at Zeitgeber time 7 (ZT7) in SD, and ZT15 in LD, ZT0 marking the transition from dark to light. For leaf growth analysis, individual leaves were harvested at different time points, flattened on white paper and then digitally scanned. Leaf areas (blade and petiole) were calculated from the binary images using ImageJ software (http://rsb.info.nih.gov/ij/). Leaves from 10 to 15 plants were analyzed.

\section{Ploidy analysis}

Leaves 1 to 4 were harvested, chopped with a razor blade in $800 \mu \mathrm{l}$ of Galbraith buffer, filtered over a $30 \mu \mathrm{m}$ mesh, and $150 \mu \mathrm{l}$ of a propidium iodide solution $(100 \mu \mathrm{g} / \mathrm{ml})$ was added [97]. The quantification of the nuclear DNA content was performed on a CyFlow cytometer using the FloMax ${ }^{\circ}$ software (Sysmex Partec, France) as described [98]. The endoreduplication index was calculated by using the formula: $\mathrm{EI}=0 \mathrm{x}(\%$ of $2 \mathrm{C})+$ $1 x(\%$ of $4 C)+2 x(\%$ of $8 C)+3 x(\%$ of $16 C)+4 x(\%$ of $32 \mathrm{C})$.

\section{Expression analysis}

Total RNAs were prepared from rosette material, treated and reverse transcribed, as previously described [99]. Quantitative real-time PCR was performed on a BioRad CFX96 apparatus using the SYBR green Master Mix (BioRad) following manufacturer's instructions. UBIQUITIN10 was used as reference gene. Primers are listed in Additional file 22. For GUS histochemical staining, plants were collected in the staining solution $(1 \mathrm{mM}$ X-Gluc (5-bromo-4-chloro-3-indolyl-ß-D-glucuronide), $0.1 \mathrm{M}$ sodium phosphate buffer, $\mathrm{pH} 7.0,2 \mathrm{mM}$ potassium ferrocyanide, $2 \mathrm{mM}$ potassium ferricyanide, and $0.5 \%$ Triton X-100), infiltrated under vacuum 3 times, for 5 min each, and incubated at $37^{\circ} \mathrm{C}$ overnight. Samples were then washed in $70 \%$ ethanol and observed under a light microscope.

\section{RNA extraction, library preparation and sequencing}

Total RNA was extracted with the Plant RNeasy Mini kit (QIAGEN). $10 \mu \mathrm{g}$ of RNA was treated with TURBO DNA-free kit (Ambion Ref. AM1907) and cleaned-up from enzymatic reactions with RNeasy 
MinElute Cleanup Kit (QIAGEN Ref. 74,204), following the manufacture instructions. RNA integrity and concentration were analyzed with the Agilent 2100 Bioanalyzer and the Agilent RNA 6000 Nano Kit (Ref. 5067-1511). For one replicate, leaves 3-4 were dissected from 20 plants and pooled. Three independent replicates were performed for each time point. Strand specific sequencing libraries were prepared from polyA RNAs using the Illumina Tru-Seq stranded RNA sample preparation v2 kit. Four libraries were multiplexed per lane and paired-end (PE) sequenced on an Illumina HiSeq 2000. Over 40 millions of 150 bp reads were generated per sample. All steps of the experiment, from growth conditions to bioinformatic analyses, were recorded in CATdb database [100] (http://tools.ips2.u-psud.fr/CATdb/) Project ID NGS2015_01_Transition according to the international standard MINSEQE minimum information about a high-throughput sequencing experiment.

\section{RNA-Seq data analysis}

RNA-Seq samples were processed using the following pipeline: the read pre-processing criteria included trimming library adapters and performing quality control checks using FastQC. The raw data (fastq) were trimmed using the FastX toolkit (Phred Quality Score $>20$, read length $>30$ bases). The Bowtie 2 mapper [101] was used to align reads against the $A$. thaliana TAIR 10 transcriptome. On average, 99\% passed the quality filter and were uniquely mapped to the TAIR 10 reference genome. We extracted 33,602 genes from TAIR10 version database [102] with one isoform per gene corresponding to the representative gene model (longest coding sequence) given by TAIR10. The abundance of each gene was calculated by a local script, which parses SAM files and counts only paired-end reads for which both reads map unambiguously one gene, and by removing multi-hits. According to these rules, around 96\% of PE reads were associated with a gene, $2 \%$ PE reads unmapped and $2 \%$ of PE reads with multi-hits were removed.

For differential expression analysis, we discarded genes, which did not have at least 1 read after a count per million (CPM) normalization, in at least one half of the samples. The library sizes were normalized using the TMM method. The count distribution was modelled with a negative binomial Generalized Linear Model (GLM) where the harvest date was considered. Dispersion was estimated by the edgeR method [103] in the statistical software ' $\mathrm{R}$ ' ( $\mathrm{R}$ Core team, 2015). The $p$-values were adjusted by the Benjamini-Hochberg procedure to control FDR. A gene was differentially expressed when its adjusted p-value was lower than 0.05 .

\section{Analysis of IncRNAs}

We gathered a non-redundant dataset (IJPB_lncDB) of 14,621 putative IncRNA sequences from published lncRNAs datasets [26, 104-106]. Redundant information was removed. Datasets were organized into three subsets according to strand information (+, -, Not Available (NA)). For each subset, we merged overlapping or "book-ended" IncRNA in a single transcription unit (lncTU). Three FASTA files of lncTUs were established: one with 5055 TUs on the positive strand, another one with 4851 putative TUs on the negative strand, and the last one with 4715 TUs, without strand information. All reads were mapped against the IJPB_lncDB using the Bowtie 2 mapper [107] using the same count criteria. Whereas $96 \%$ of the paired-end reads mapped to the TAIR10 genome as expected, the mean mapping percentage to the lncRNA dataset was $0.78 \%$. To establish the differentially expressed lncTUs, we used the GLM of edgeR, without or with filter using either the Bonferroni or Benjamini-Hochberg (BH) test corrections. All lncTUs differentially expressed identified using Bonferroni test were present in the list of DE lncTUs identified using $\mathrm{BH}$ test. We further analyzed $\mathrm{BH}$ DE lncTUs. No bias was observed for the distribution of the DE-lncRNAs on the two strands.

To determine overlaps between lncRNA and annotated chromatin states we used the online BEDTools suite. We established intersects for all lncRNAs, the DE IncRNAs and randomly reshuffled regions of identical size to compute the fold changes between observed and randomly distributed IncRNAs. The hierarchical clustering analysis was performed using the Multiexperiment Viewer tool (MeV 4_8) with the average linkage method, gene leaf order optimization and Pearson correlations.

\section{Model for the co-expression analysis}

Co-expression analysis was carried out on differentially expressed transcripts and lncRNAs using the $\mathrm{R}$ package coseq [108] (https://bioconductor.org/packages/devel/bioc/vignettes/coseq/inst/doc/coseq.html).

We ran two clustering methods ( $K$-means algorithm and Gaussian mixture models) for two different count data transformation functions (the centred log ratio (CLR) and $\log$ CLR for $K$-means; Logit and arcsin for Gaussian mixture models). Ten technical replicates were performed for each combination of method/ transformation to prevent initialization problems. We computed 30 models from $K=10$ to $K=40$ ( $K=$ number of clusters). For each method, the best $K$ was selected via the slope heuristics approach for $K$-means methods or via the Integrated Completed Likelihood (ICL) criterion for Gaussian mixture models. The transformation function, which minimizes the within clusters variability (for $K$-means algorithm) or the ICL 
criterion (for Gaussian mixture models) was retained. Since the $K$-means algorithm seemed more sensitive to extreme expression data, we finally retained the Gaussian mixture model method with the arcsin transformation function and $\mathrm{K}=24$. This method provided a more homogeneous number of transcripts per cluster.

For each cluster, a Singular Enrichment Analysis (SEA) of GO terms (AgriGO v2.0) [109] was performed (Fisher test with a FDR cut-off at 0.01 and a minimum number of mapping entries of 10), using a customized reference corresponding to expressed genes during the time course experiment. An heatmap comparing the results of individual cluster's SEA were obtained using the SEACOMPARE program (AgriGO v2.0).

\section{Bioinformatics analysis}

We extracted 413 genes (FLGs) from FLOR-ID [16], comprising the 306 core flowering time genes, genes involved in flower meristem identity and flower development (FMI-FD) and pending annotated flowering time genes. For the analysis of the TFs we used the PlantTFDB 4.0 (http://planttfdb.cbi.pku.edu.cn/). Chromatin-associated genes were described in [38]. Hierarchical clustering was performed using the Multiple Experiment Viewer tool $(\mathrm{MeV})$ with the Pearson correlation metric and average linkage clustering as linkage method [110]. We performed functional annotation and classification using the "AgriGO" Gene Ontology tool [109] and the Classification SuperViewer Tool from BAR [111]. For each cluster, we extracted the biological process (BP) GO terms with the best FDR and the best specialized and enriched "child" GO terms (Additional file 23). Venn diagrams were generated using the online tool provided by T. Hulsen (http:// bioinformatics.psb.ugent.be/webtools/Venn/).

\section{Motif detection}

The "Preferentially Located Motifs" algorithm is based on the over-representation of a motif around the Transcription Start Site (TSS), region -300 from TSS to 5'UTR, compared to its distribution in the region of 1000 to -300 (learning region) before the TSS [46]. We also explored a list of 419 motifs merged from PLACE [112] and AGRIS (http://agris-knowledgebase.org/ AtcisDB/bindingsites.html) to find enrichment ( $p$-value $<0.05$ ) around the TSS compared to all Arabidopsis genome (Additional file 23).

\section{Accession number}

The accession number into the international GEO repository is GSE116123.

\section{Additional files}

Additional file 1: Figure S1. Characterization of the SD-LD switch. (a) Flowering time according to the number of weeks in SD. Rosette and cauline leaves were recorded on plants when the first flowers appeared. The bolting time was quantified when the stem was $0.5 \mathrm{~cm}$ high from sowing. Three biological replicates were performed with 12 plants, each. (b) Expression of the AP1::GUS reporter gene in the apical shoots of plants grown 4 weeks in SD, and then transferred to LD. Day after transfer (dat). (PDF 772 kb)

Additional file 2: Figure S2. Growth of rosette leaves in response to SD or a SD-LD switch. (a) Measurements of the total rosette leaf areas. (b) Area measurement of the first six leaves. Col-0 plants were grown in SD for 4 weeks, then kept in SD (continuous line) or transferred in LD (dash line). Two biological replicates were performed with 10 plants, each. Experimental values are mean \pm SEM. (PDF $70 \mathrm{~kb}$ )

Additional file 3: Figure S3. RNA-Seq experiments and expressed gene distributions. (a) Library sizes of the biological replicates. (b) Read mapping for the different time points and biological replicates. (c) Distribution of the expressed genes in the genomes. (d) Differentially expressed genes (DEGs) and Venn diagram with the three main comparisons. (e) Distribution of the expressed genes in the main gene classes. (PDF $194 \mathrm{~kb}$ )

Additional file 4: Figure S4. Analysis of IncRNAs. (a) Resources used to construct the IncTU dataset. (b) IncTUs differentially expressed for each comparison using the Benjamini-Hochberg (BH) test. (PDF $51 \mathrm{~kb}$ )

Additional file 5: List of IncTUs. (XLSX 639 kb)

Additional file 6: List of genes per cluster. (XLSX $187 \mathrm{~kb}$ )

Additional file 7: Best enriched biological process (BP) GO terms for the 24 clusters. The best BP GO term was extracted with its FDR from each SEA analysis. (XLSX $11 \mathrm{~kb}$ )

Additional file 8: Figure S5. Differentially expressed endoreduplicationrelated genes extracted from the ThaleMine database. Log2 (Fold-Change) is reported. In black, non-statistically significant fold change values. (PDF $44 \mathrm{~kb}$ )

Additional file 9: Distributions of differentially expressed genes of the FLOR-ID database in the clusters. (XLSX $28 \mathrm{~kb}$ )

Additional file 10: Hormone-related genes supported by genetic evidence in clusters. (XLSX $22 \mathrm{~kb}$ )

Additional file 11: Figure S6. Floral transition and hormone pathways. (a) Distribution of AHD genes in clusters. (b) Genes associated with gibberellin metabolism and responses. (PDF $56 \mathrm{~kb}$ )

Additional file 12: Hormone-related genes involved in several hormonal pathways in the clusters. (XLSX $10 \mathrm{~kb}$ )

Additional file 13: Motifs identified with the PLM algorithm. (XLSX 87 kb)

Additional file 14: Distribution of the TFs among the clusters. (XLSX 38 $\mathrm{kb})$

\section{Additional file 15: TFs from the FLOR-ID. (XLSX $12 \mathrm{~kb}$ )}

Additional file 16: Figure S7. Screenshots of the TF2network user interface using the 64 TFs differentially regulated and belonging to FLORID. The three Cytoscape panels show the gene regulatory networks with the first 5 best-ranked regulators (blue diamonds). Green diamonds represent TFs and green circles, non-TF genes, according to the TF2Network interface annotations. The dashed arrows indicated PWM motifs for the corresponding regulators. The blue lines indicate protein-protein interactions. (PDF 524 kb)

Additional file 17: Differentially expressed genes associated with chromatin biology. (XLSX $19 \mathrm{~kb}$ )

Additional file 18: Distribution of differentially expressed genes associated with chromatin biology. CR: chromatin remodeling. (XLSX 9 $\mathrm{kb})$

Additional file 19: Expression of IncTUs and their NATs. (XLSX $25 \mathrm{~kb}$ ) 
Additional file 20: Figure S8. Expression of MAF5 and its antisense IncTU located in the $3^{\prime}$ end of MAF5 region at TO, T2 and T3. (a) Fold changes. (b) Browser snapshot showing the expression profiles. BR1: biological replicate number 1. (PDF $77 \mathrm{~kb}$ )

Additional file 21: Distribution of the TFBFs in the regions of the IncTUs. (XLSX $21 \mathrm{~kb}$ )

Additional file 22: List of oligonucleotides. (XLSX $8 \mathrm{~kb}$ )

Additional file 23: Information in PLACE and AGRIS databases on the identified motifs. (XLSX $38 \mathrm{~kb}$ )

\section{Abbreviations}

DEG: Differentially expressed gene; FT: Transcription factor; GO: Gene ontology; LD: Long days; Inc: Long non-coding; SD: Short days; TU: Transcription unit; ZT: Zeitgeber

\section{Acknowledgements}

We thank Bruno Letarnec and Hervé Ferry for plant care in the greenhouses. We thank Prof. Dr. Gerco Angenent and Dr. Suraj Jamge for the seeds of the API::GUS transgenic line (unpublished material) in the frame of the ITN Project EpiTRAITS. We are very grateful to Dr. Ortrun Mittelsten Scheid and the VBCF platform for preliminary sequencing tests. We thank Johanne Thevenin and the plant cytology and imaging platform (PCIV)" of the IJPB Plant Observatory for the technical support on flow cytometry. We are very grateful to Dr. Jeffrey Leung for careful reading and comments. We are very grateful to and thank Prof. Dr. Ronald E. Koes (Amsterdam University) for providing SDP a PhD short-term fellowship from the University of Amsterdam. The funding of SDP is gratefully acknowledged.

\section{Funding}

SDP was supported by a PhD fellowship provided by the European Commission Seventh Framework-People-2012-ITN Project EpiTRAITS (no-316965), a short-term INRA grant and by a PhD short-term fellowship from the Swammerdam Institute for Life Sciences of the University of Amsterdam. The sequencing platform (POPSIPS2) and the IJPB benefit from the support of the LabEx Saclay Plant Sciences-SPS (ANR-10-LABX-0040-SPS). The funding agencies played no role in the design of the study and collection, analysis, and interpretation of data and in writing the manuscript.

\section{Availability of data and materials}

The datasets used and analyzed during the current study are available from the corresponding author on reasonable request.

\section{Authors' contributions}

SDP performed experiments and analyses. LST performed libraries and sequencing. $A M, D C, N B, C G, V B$, and $F G$ performed bioinformatics analyses. VG analyzed data and coordinated the analyses. SDP, PF and VG wrote the manuscript. All authors read and approved the final manuscript.

\section{Ethics approval and consent to participate}

The experimental research on plants performed in this study complies with institutional, national and international guidelines.

\section{Consent for publication}

Not applicable.

\section{Competing interests}

The authors declare that they have no competing interests.

\section{Publisher's Note}

Springer Nature remains neutral with regard to jurisdictional claims in published maps and institutional affiliations.

\section{Author details}

'Institut Jean-Pierre Bourgin, INRA, AgroParisTech, CNRS, Université Paris-Saclay, INRA Centre de Versailles-Grignon, Bât. 2, RD10 Route de Saint-Cyr, 78000 Versailles, France. ${ }^{2}$ Institute of Plant Sciences Paris-Saclay (IPS2), CNRS, INRA, Université Paris-Sud, Université Evry, Université Paris-Saclay, Bâtiment 630, Plateau du Moulon, 91192 Gif-sur-Yvette, France. ${ }^{3}$ Institute of Plant Sciences Paris-Saclay (IPS2), CNRS, INRA, Université Paris
Diderot, Sorbonne Paris-Cité, Bâtiment 630, Plateau du Moulon, 91192 Gif-sur-Yvette, 91405 Orsay, France. ${ }^{4}$ Swammerdam Institute for Life Sciences, University of Amsterdam, 1098XH Amsterdam, The Netherlands.

Received: 4 October 2018 Accepted: 24 March 2019

Published online: 11 April 2019

\section{References}

1. Hepworth J, Dean C. Flowering Locus C's lessons: conserved chromatin switches underpinning developmental timing and adaptation. Plant Physiol. 2015;168(4):1237-45.

2. Pajoro A, Biewers S, Dougali E, Leal Valentim F, Mendes MA, Porri A, Coupland G, Van de Peer Y, van Dijk AD, Colombo L, et al. The (r)evolution of gene regulatory networks controlling Arabidopsis plant reproduction: a two-decade history. J Exp Bot. 2014;65(17):4731-45.

3. Putterill J, Robson F, Lee K, Simon R, Coupland G. The CONSTANS gene of Arabidopsis promotes flowering and encodes a protein showing similarities to zinc finger transcription factors. Cell. 1995;80:847-57.

4. Bernier $\mathrm{G}$, Perilleux C. A physiological overview of the genetics of flowering time control. Plant Biotechnol J. 2005:3(1):3-16.

5. Song YH, Shim JS, Kinmonth-Schultz HA, Imaizumi T. Photoperiodic flowering: time measurement mechanisms in leaves. Annu Rev Plant Biol. 2015;66:441-64

6. Corbesier L, Vincent C, Jang S, Fornara F, Fan Q, Searle I, Giakountis A, Farrona S, Gissot L, Turnbull C, et al. FT protein movement contributes to long-distance signaling in floral induction of Arabidopsis. Science. 2007; 316(5827):1030-3.

7. Corbesier L, Gadisseur I, Silvestre G, Jacqmard A, Bernier G. Design in Arabidopsis thaliana of a synchronous system of floral induction by one long day. Plant J. 1996;9(6):947-52.

8. McClung CR, Lou P, Hermand V, Kim JA. The importance of ambient temperature to growth and the induction of flowering. Frontiers Plant Sci. 2016:7:1266.

9. Bratzel F, Turck F. Molecular memories in the regulation of seasonal flowering: from competence to cessation. Genome Biol. 2015;16:192.

10. Conti L. Hormonal control of the floral transition: can one catch them all? Dev Biol. 2017:430(2):288-301.

11. Mahrez W, Shin J, Munoz-Viana R, Figueiredo DD, Trejo-Arellano MS, Exner V. Siretskiy A, Gruissem W, Kohler C, Hennig L. BRR2a affects flowering time via FLC splicing. PLoS Genet. 2016;12(4):e1005924.

12. Kwak JS, Son GH, Song JT, Seo HS. Post-translational modifications of FLOWERING LOCUS C modulate its activity. J Exp Bot. 2017;68(3):383-9.

13. Whittaker C, Dean C. The FLC Locus: a platform for discoveries in epigenetics and adaptation. Annu Rev Cell Dev Biol. 2017;33:555-75.

14. Duan HC, Wei LH, Zhang C, Wang Y, Chen L, Lu Z, Chen PR, He C, Jia G ALKBH10B is an RNA N(6)-Methyladenosine demethylase affecting Arabidopsis floral transition. Plant Cell. 2017;29(12):2995-3011.

15. Berry S, Dean C. Environmental perception and epigenetic memory: mechanistic insight through FLC. Plant J. 2015;83(1):133-48.

16. Bouche F, Lobet G, Tocquin P, Perilleux C. FLOR-ID: an interactive database of flowering-time gene networks in Arabidopsis thaliana. Nucleic Acids Res. 2016:44(D1):D1167-71.

17. Yamada M. Functions of long intergenic non-coding (linc) RNAs in plants. J Plant Res. 2017;130(1):67-73.

18. Krzyczmonik K, Wroblewska-Swiniarska A, Swiezewski S. Developmental transitions in Arabidopsis are regulated by antisense RNAs resulting from bidirectionally transcribed genes. RNA Biol. 2017;14(7):838-42.

19. Kim DH, Xi Y, Sung S. Modular function of long noncoding RNA, COLDAIR, in the vernalization response. PLoS Genet. 2017;13(7):e1006939.

20. Heo JB, Sung S. Vernalization-mediated epigenetic silencing by a long intronic noncoding RNA. Science. 2011;331(6013):76-9.

21. Swiezewski S, Liu F, Magusin A, Dean C. Cold-induced silencing by long antisense transcripts of an Arabidopsis Polycomb target. Nature. 2009; 462(7274):799-802.

22. Liu F, Marquardt S, Lister C, Swiezewski S, Dean C. Targeted 3' processing of antisense transcripts triggers Arabidopsis FLC chromatin silencing. Science. 2010:327(5961):94-7.

23. Shin JH, Chekanova JA. Arabidopsis RRP6L1 and RRP6L2 function in FLOWERING LOCUS C silencing via regulation of antisense RNA synthesis. PLoS Genet. 2014;10(9):e1004612. 
24. Henriques R, Wang H, Liu J, Boix M, Huang LF, Chua NH. The antiphasic regulatory module comprising CDF5 and its antisense RNA FLORE links the circadian clock to photoperiodic flowering. New Phytol. 2017;216(3):854-67.

25. Wang KC, Chang HY. Molecular mechanisms of long noncoding RNAs. Mol Cell. 2011:43(6):904-14.

26. Di C, Yuan J, Wu Y, Li J, Lin H, Hu L, Zhang T, Qi Y, Gerstein MB, Guo Y, et al. Characterization of stress-responsive IncRNAs in Arabidopsis thaliana by integrating expression, epigenetic and structural features. The Plant J. 2014; 80(5):848-61.

27. Yuan C, Wang J, Harrison AP, Meng X, Chen D, Chen M. Genome-wide view of natural antisense transcripts in Arabidopsis thaliana. DNA Res. 2015;22(3): 233-43.

28. Bouche F, D'Aloia M, Tocquin P, Lobet G, Detry N, Perilleux C. Integrating roots into a whole plant network of flowering time genes in Arabidopsis thaliana. Sci Rep. 2016;6:29042.

29. Klepikova AV, Logacheva MD, Dmitriev SE, Penin AA. RNA-seq analysis of an apical meristem time series reveals a critical point in Arabidopsis thaliana flower initiation. BMC Genomics. 2015;16:466.

30. Torti S, Fornara F, Vincent C, Andres F, Nordstrom K, Gobel U, Knoll D, Schoof $\mathrm{H}$, Coupland $\mathrm{G}$. Analysis of the Arabidopsis shoot meristem transcriptome during floral transition identifies distinct regulatory patterns and a leucine-rich repeat protein that promotes flowering. Plant Cell. 2012 24(2):444-62.

31. Schmid M, Uhlenhaut NH, Godard F, Demar M, Bressan R, Weigel D, Lohmann JU. Dissection of floral induction pathways using global expression analysis. Development. 2003;130(24):6001-12.

32. You Y, Sawikowska A, Neumann M, Pose D, Capovilla G, Langenecker T, Neher RA, Krajewski P, Schmid M. Temporal dynamics of gene expression and histone marks at the Arabidopsis shoot meristem during flowering. Nat Commun. 2017:8:15120.

33. Samach A, Onouchi H, Gold SE, Ditta GS, Schwarz-Sommer Z, Yanofsky MF, Coupland G. Distinct roles of CONSTANS target genes in reproductive development of Arabidopsis. Science. 2000;288(5471):1613-6.

34. Wigge PA, Kim MC, Jaeger KE, Busch W, Schmid M, Lohmann JU, Weigel D. Integration of spatial and temporal information during floral induction in Arabidopsis. Science. 2005;309(5737):1056-9.

35. Michaels SD, Himelblau E, Kim SY, Schomburg FM, Amasino RM. Integration of flowering signals in winter-annual Arabidopsis. Plant Physiol. 2005;137(1): 149-56.

36. Yamaguchi A, Kobayashi Y, Goto K, Abe M, Araki T. TWIN SISTER OF FT (TSF) acts as a floral pathway integrator redundantly with FT. Plant Cell Physiol. 2005:46(8):1175-89.

37. Somssich M, Khan GA, Persson S. Cell Wall heterogeneity in root development of Arabidopsis. Front Plant Sci. 2016;7:1242.

38. Chupeau MC, Granier F, Pichon O, Renou JP, Gaudin V, Chupeau Y. Characterization of the early events leading to totipotency in an Arabidopsis protoplast liquid culture by temporal transcript profiling. Plant Cell. 2013;25(7):2444-63.

39. Kalve S, De Vos D, Beemster GT. Leaf development: a cellular perspective. Front Plant Sci. 2014;5:362

40. Dewitte W, Riou-Khamlichi C, Scofield S, Healy JM, Jacqmard A, Kilby NJ, Murray JA. Altered cell cycle distribution, hyperplasia, and inhibited differentiation in Arabidopsis caused by the D-type cyclin CYCD3. Plant Cell. 2003;15(1):79-92.

41. Imai KK, Ohashi Y, Tsuge T, Yoshizumi T, Matsui M, Oka A, Aoyama T. The Atype cyclin CYCA2;3 is a key regulator of ploidy levels in Arabidopsis endoreduplication. Plant Cell. 2006;18(2):382-96.

42. Jiang Z, Liu X, Peng Z, Wan Y, Ji Y, He W, Wan W, Luo J, Guo H. AHD2.0: an update version of Arabidopsis hormone database for plant systematic studies. Nucleic Acids Res. 2011;39(Database issue):D1123-9.

43. Shu K, Chen Q, Wu Y, Liu R, Zhang H, Wang S, Tang S, Yang W, Xie Q. ABSCISIC ACID-INSENSITIVE 4 negatively regulates flowering through directly promoting Arabidopsis FLOWERING LOCUS C transcription. J Exp Bot. 2016;67(1):195-205.

44. Zhang J, Vankova R, Malbeck J, Dobrev PI, Xu Y, Chong K, Neff MM AtSOFL1 and AtSOFL2 act redundantly as positive modulators of the endogenous content of specific cytokinins in Arabidopsis. PLoS One. 2009; 4(12):e8236.

45. Argyros RD, Mathews DE, Chiang $\mathrm{YH}$, Palmer CM, Thibault DM, Etheridge $\mathrm{N}$, Argyros DA, Mason MG, Kieber JJ, Schaller GE. Type B response regulators of
Arabidopsis play key roles in cytokinin signaling and plant development. Plant Cell. 2008;20(8):2102-16.

46. Bernard V, Brunaud V, Lecharny A. TC-motifs at the TATA-box expected position in plant genes: a novel class of motifs involved in the transcription regulation. BMC Genomics. 2010;11:166

47. Gupta S, Stamatoyannopoulos JA, Bailey TL, Noble WS. Quantifying similarity between motifs. Genome Biol. 2007:8(2):R24.

48. Franco-Zorrilla JM, Lopez-Vidriero I, Carrasco JL, Godoy M, Vera P, Solano R. DNA-binding specificities of plant transcription factors and their potential to define target genes. Proc Natl Acad Sci U S A. 2014;111(6):2367-72.

49. O'Malley RC, Huang SC, Song L, Lewsey MG, Bartlett A, Nery JR, Galli M, Gallavotti A, Ecker JR. Cistrome and Epicistrome features shape the regulatory DNA landscape. Cell. 2016;165(5):1280-92.

50. Michael TP, McClung CR. Phase-specific circadian clock regulatory elements in Arabidopsis. Plant Physiol. 2002;130(2):627-38.

51. Wang H, Li Y, Pan J, Lou D, Hu Y, Yu D. The bHLH transcription factors MYC2, MYC3, and MYC4 are required for Jasmonate-mediated inhibition of flowering in Arabidopsis. Mol Plant. 2017;10(11):1461-4.

52. Molitor A, Latrasse D, Zytnicki M, Andrey P, Houba-Herin N, Hachet M, Battail C, Del Prete S, Alberti A, Quesneville H, et al. The Arabidopsis hnRNPQ protein LIF2 and the PRC1 subunit LHP1 function in concert to regulate the transcription of stress-responsive genes. Plant Cell. 2016;28:2197-211.

53. Gaudin V, Libault M, Pouteau S, Juul T, Zhao G, Lefebvre D, Grandjean O. Mutations in LIKE HETEROCHROMATIN PROTEIN 1 affect flowering time and plant architecture in Arabidopsis. Devevlopment. 2001;128:4847-58.

54. Zhang X, Germann S, Blus BJ, Khorasanizadeh S, Gaudin V, Jacobsen SE. The Arabidopsis LHP1 protein colocalizes with histone H3 Lys27 trimethylation. Nat Struct Mol Biol. 2007;14(9):869-71.

55. Zhang B, Wang L, Zeng L, Zhang C, Ma H. Arabidopsis TOE proteins convey a photoperiodic signal to antagonize CONSTANS and regulate flowering time. Genes Dev. 2015;29(9):975-87.

56. Jin J, Tian F, Yang DC, Meng YQ, Kong L, Luo J, Gao G. PlantTFDB 4.0: toward a central hub for transcription factors and regulatory interactions in plants. Nucleic Acids Res. 2017:45(D1):D1040-5.

57. Gangappa SN, Botto JF. The BBX family of plant transcription factors. Trends Plant Sci. 2014;19(7):460-70.

58. Khanna R, Kronmiller B, Maszle DR, Coupland G, Holm M, Mizuno T, Wu SH. The Arabidopsis B-box zinc finger family. Plant Cell. 2009;21(11):3416-20.

59. Cheng XF, Wang ZY. Overexpression of COL9, a CONSTANS-LIKE gene, delays flowering by reducing expression of $\mathrm{CO}$ and $\mathrm{FT}$ in Arabidopsis thaliana. Plant J. 2005;43(5):758-68.

60. Hassidim M, Harir Y, Yakir E, Kron I, Green RM. Over-expression of CONSTANS-LIKE 5 can induce flowering in short-day grown Arabidopsis. Planta. 2009;230(3):481-91.

61. Zhao H, Wu D, Kong F, Lin K, Zhang H, Li G. The Arabidopsis thaliana nuclear factor $Y$ transcription factors. Front Plant Sci. 2016;7:2045.

62. Swain S, Myers ZA, Siriwardana CL, Holt BF 3rd. The multifaceted roles of NUCLEAR FACTOR-Y in Arabidopsis thaliana development and stress responses. Biochim Biophys Acta. 2017;1860(5):636-44.

63. Kumimoto RW, Zhang Y, Siefers N, Holt BF 3rd. NF-YC3, NF-YC4 and NF-YC9 are required for CONSTANS-mediated, photoperiod-dependent flowering in Arabidopsis thaliana. Plant J. 2010;63(3):379-91.

64. Kumimoto RW, Adam L, Hymus GJ, Repetti PP, Reuber TL, Marion CM Hempel FD, Ratcliffe OJ. The nuclear factor Y subunits NF-YB2 and NF-YB3 play additive roles in the promotion of flowering by inductive long-day photoperiods in Arabidopsis. Planta. 2008;228(5):709-23.

65. Foyer $\mathrm{CH}$, Karpinska B, Krupinska K. The functions of WHIRLY1 and REDOXRESPONSIVE TRANSCRIPTION FACTOR 1 in cross tolerance responses in plants: a hypothesis. Philos Trans R Soc Lond B Biol Sci. 2014;369(1640): 20130226.

66. Cai Q, Guo L, Shen ZR, Wang DY, Zhang Q, Sodmergen. Elevation of pollen mitochondrial DNA copy number by WHIRLY2: altered respiration and pollen tube growth in Arabidopsis. Plant Physiol. 2015;169(1):660-73.

67. Kulkarni SR, Vaneechoutte D, Van de Velde J, Vandepoele K. TF2Network: predicting transcription factor regulators and gene regulatory networks in Arabidopsis using publicly available binding site information. Nucleic Acids Res. 2018;46(6):e31.

68. Sequeira-Mendes J, Araguez I, Peiro R, Mendez-Giraldez R, Zhang X Jacobsen SE, Bastolla U, Gutierrez C. The functional topography of the Arabidopsis genome is organized in a reduced number of linear motifs of chromatin states. Plant Cell. 2014;26(6):2351-66. 
69. Xing D, Wang Y, Xu R, Ye X, Yang D, Li QQ. The regulatory role of Pcf11similar-4 (PCFS4) in Arabidopsis development by genome-wide physical interactions with target loci. BMC Genomics. 2013;14:598.

70. Gregis V, Andres F, Sessa A, Guerra RF, Simonini S, Mateos JL, Torti S Zambelli F, Prazzoli GM, Bjerkan KN, et al. Identification of pathways directly regulated by SHORT VEGETATIVE PHASE during vegetative and reproductive development in Arabidopsis. Genome Biol. 2013;14(6):R56.

71. Immink RG, Pose D, Ferrario S, Ott F, Kaufmann K, Valentim FL, de Folter $\mathrm{S}$, van der Wal F, van Dijk AD, Schmid M, et al. Characterization of SOC1's central role in flowering by the identification of its upstream and downstream regulators. Plant Physiol. 2012;160(1):433-49.

72. Mateos JL, Madrigal P, Tsuda K, Rawat V, Richter R, Romera-Branchat M, Fornara F, Schneeberger K, Krajewski P, Coupland G. Combinatorial activities of SHORT VEGETATIVE PHASE and FLOWERING LOCUS C define distinct modes of flowering regulation in Arabidopsis. Genome Biol. 2015;16:31.

73. Deng W, Ying H, Helliwell CA, Taylor JM, Peacock WJ, Dennis ES. FLOWERING LOCUS C (FLC) regulates development pathways throughout the life cycle of Arabidopsis. Proc Natl Acad Sci U S A. 2011;108(16):6680-5.

74. Tao Z, Shen L, Liu C, Liu L, Yan Y, Yu H. Genome-wide identification of SOC1 and SVP targets during the floral transition in Arabidopsis. Plant J. 2012;70(4):549-61.

75. Corbesier $L$, Lejeune $P$, Bernier $G$. The role of carbohydrates in the induction of flowering in Arabidopsis thaliana: comparison between the wild type and a starchless mutant. Planta. 1998;206(1):131-7.

76. King RW, Hisamatsu T, Goldschmidt EE, Blundell C. The nature of floral signals in Arabidopsis. I. Photosynthesis and a far-red photoresponse independently regulate flowering by increasing expression of FLOWERING LOCUS T (FT). J Exp Bot. 2008;59(14):3811-20.

77. Ortiz-Marchena MI, Romero JM, Valverde F. Photoperiodic control of sugar release during the floral transition: what is the role of sugars in the florigenic signal? Plant Signal Behav. 2015;10(5):e1017168.

78. Bernier G, Havelange A, Houssa C, Petitjean A, Lejeune P. Physiological signals that induce flowering. Plant Cell. 1993;5(10):1147-55.

79. Sonderby IE, Geu-Flores F, Halkier BA. Biosynthesis of glucosinolates--gene discovery and beyond. Trends Plant Sci. 2010;15(5):283-90.

80. Mohammadin S, Nguyen TP, van Weij MS, Reichelt M, Schranz ME. Flowering Locus C (FLC) is a potential major regulator of Glucosinolate content across developmental stages of Aethionema arabicum (Brassicaceae). Frontiers Plant Sci. 2017;8:876.

81. Pruneda-Paz JL, Breton G, Para A, Kay SA. A functional genomics approach reveals $\mathrm{CHE}$ as a component of the Arabidopsis circadian clock. Science. 2009;323(5920):1481-5.

82. Wang Y, Li L, Ye T, Lu Y, Chen X, Wu Y. The inhibitory effect of ABA on floral transition is mediated by ABI5 in Arabidopsis. J Exp Bot. 2013;64(2):675-84.

83. Shu K, Luo X, Meng Y, Yang W. Toward a molecular understanding of abscisic acid actions in floral transition. Plant Cell Physiol. 2018;59(2):215-21.

84. Riboni M, Galbiati M, Tonelli C, Conti L. GIGANTEA enables drought escape response via abscisic acid-dependent activation of the florigens and SUPPRESSOR OF OVEREXPRESSION OF CONSTANS. Plant Physiol. 2013; 162(3):1706-19.

85. Riboni M, Robustelli Test A, Galbiati M, Tonelli C, Conti L. ABA-dependent control of GIGANTEA signalling enables drought escape via up-regulation of FLOWERING LOCUS T in Arabidopsis thaliana. J Exp Bot. 2016;67(22):6309_ 22.

86. Jacamard A, Gadisseur I, Bernier G. Cell division and morphological changes in the shoot apex of Arabidopsis thaliana during floral transition. Ann Bot. 2003:91(5):571-6.

87. Dewitte W, Scofield S, Alcasabas AA, Maughan SC, Menges M, Braun N, Collins C, Nieuwland J, Prinsen E, Sundaresan V, et al. Arabidopsis CYCD3 Dtype cyclins link cell proliferation and endocycles and are rate-limiting for cytokinin responses. Proc Natl Acad Sci U S A. 2007;104(36):14537-42.

88. Bao Z, Yang H, Hua J. Perturbation of cell cycle regulation triggers plant immune response via activation of disease resistance genes. Proc Natl Acad Sci U S A. 2013;110(6):2407-12

89. Ishida T, Adachi S, Yoshimura M, Shimizu K, Umeda M, Sugimoto K. Auxin modulates the transition from the mitotic cycle to the endocycle in Arabidopsis. Development. 2010;137(1):63-71.

90. Ishida T, Fujiwara S, Miura K, Stacey N, Yoshimura M, Schneider K, Adachi S, Minamisawa K, Umeda M, Sugimoto K. SUMO E3 ligase HIGH PLOIDY2 regulates endocycle onset and meristem maintenance in Arabidopsis. Plant Cell. 2009;21(8):2284-97.
91. Cookson SJ, Radziejwoski A, Granier C. Cell and leaf size plasticity in Arabidopsis: what is the role of endoreduplication? Plant Cell Environ. 2006; 29(7):1273-83.

92. Gegas VC, Wargent JJ, Pesquet E, Granqvist E, Paul ND, Doonan JH. Endopolyploidy as a potential alternative adaptive strategy for Arabidopsis leaf size variation in response to UV-B. J Exp Bot. 2014;65(10):2757-66.

93. Fiers M, Hoogenboom J, Brunazzi A, Wennekes T, Angenent GC, Immink $\mathrm{RGH}$. A plant-based chemical genomics screen for the identification of flowering inducers. Plant Methods. 2017;13:78

94. Karimi M, Inze D, Depicker A. GATEWAY vectors for agrobacterium-mediated plant transformation. Trends Plant Sci. 2002;7(5):193-5.

95. Pouteau S, Albertini C. The significance of bolting and floral transitions as indicators of reproductive phase change in Arabidopsis. J Exp Bot. 2009; 60(12):3367-77.

96. Latrasse D, Germann S, Houba-Herin N, Dubois E, Bui-Prodhomme D, Hourcade D, Juul-Jensen T, Le Roux C, Majira A, Simoncello N, et al. Control of flowering and cell fate by LIF2, an RNA binding partner of the polycomb complex component LHP1. PLoS One. 2011;6(1):e16592.

97. Galbraith DW, Lambert GM, Macas J, Dolezel J. Analysis of nuclear DNA content and ploidy in higher plants. Curr Protoc Cytom. 2001; Chapter 7: Unit 7.6.

98. Thevenin J, Dubos C, Xu W, Le Gourrierec J, Kelemen Z, Charlot F, Nogue F, Lepiniec L, Dubreucq B. A new system for fast and quantitative analysis of heterologous gene expression in plants. New Phytol. 2012;193(2):504-12.

99. Le Roux C, Del Prete S, Boutet-Mercey S, Perreau F, Balague C, Roby D, Fagard M, Gaudin V. The hnRNP-Q protein LIF2 participates in the plant immune response. PLoS One. 2014;9(6):e99343.

100. Gagnot S, Tamby JP, Martin-Magniette ML, Bitton F, Taconnat L, Balzergue S, Aubourg S, Renou JP, Lecharny A, Brunaud V. CATdb: a public access to Arabidopsis transcriptome data from the URGV-CATMA platform. Nucleic Acids Res. 2008:36:D986-90.

101. Langmead B, Salzberg SL. Fast gapped-read alignment with bowtie 2. Nat Methods. 2012;9(4):357-9.

102. Lamesch P, Berardini TZ, Li D, Swarbreck D, Wilks C, Sasidharan R, Muller R, Dreher K, Alexander DL, Garcia-Hernandez M, et al. The Arabidopsis information resource (TAIR): improved gene annotation and new tools. Nucleic Acids Res. 2012:40(Database issue):D1202-10.

103. McCarthy DJ, Chen Y, Smyth GK. Differential expression analysis of multifactor RNA-Seq experiments with respect to biological variation. Nucleic Acids Res. 2012;40(10):4288-97.

104. Derozier S, Samson F, Tamby JP, Guichard C, Brunaud V, Grevet P, Gagnot S, Label P, Leple JC, Lecharny A, et al. Exploration of plant genomes in the FLAGdb++ environment. Plant Methods. 2011;7:8.

105. Liu J, Jung C, Xu J, Wang H, Deng S, Bernad L, Arenas-Huertero C, Chua NH. Genome-wide analysis uncovers regulation of long intergenic noncoding RNAs in Arabidopsis. Plant Cell. 2012;24(11):4333-45.

106. Xie C, Yuan J, Li H, Li M, Zhao G, Bu D, Zhu W, Wu W, Chen R, Zhao Y. NONCODEv4: exploring the world of long non-coding RNA genes. Nucleic Acids Res. 2014;42(Database issue):D98-103.

107. Langmead B, Trapnell C, Pop M, Salzberg SL. Ultrafast and memory-efficient alignment of short DNA sequences to the human genome. Genome Biol. 2009;10(3):R25.

108. Rau A, Maugis-Rabusseau C. Transformation and model choice for RNA-seq co-expression analysis. Brief bioinform. 2017;19(3):425-36.

109. Tian T, Liu Y, Yan H, You Q, Yi X, Du Z, Xu W, Su Z. agriGO v2.0: a GO analysis toolkit for the agricultural community, 2017 update. Nucleic Acids Res. 2017:45(W1):W122-W129.

110. Saeed Al, Sharov V, White J, Li J, Liang W, Bhagabati N, Braisted J, Klapa M, Currier T, Thiagarajan M, et al. TM4: a free, open-source system for microarray data management and analysis. Biotechniques. 2003;34(2):374-8.

111. Provart NJ, Gil P, Chen W, Han B, Chang HS, Wang X, Zhu T. Gene expression phenotypes of Arabidopsis associated with sensitivity to low temperatures. Plant Physiol. 2003;132(2):893-906.

112. Higo K, Ugawa Y, Iwamoto M, Korenaga T. Plant cis-acting regulatory DNA elements (PLACE) database: 1999. Nucleic Acids Res. 1999:27(1):297-300. 